\title{
The Ultraviolet-Bright, Slowly Declining Transient PS1-11af as a Partial Tidal Disruption Event.
}

\section{Citation}

Chornock, R., E. Berger, S. Gezari, B. A. Zauderer, A. Rest, L. Chomiuk, A. Kamble, et al. 2013. "THE ULTRAVIOLET-BRIGHT, SLOWLY DECLINING TRANSIENT PS1-11 af AS A PARTIAL TIDAL DISRUPTION EVENT." The Astrophysical Journal 780 (1) (December 11): 44. doi:10.1088/0004-637x/780/1/44.

\section{Published Version}

doi::10.1088/0004-637X/780/1/44

\section{Permanent link}

http://nrs.harvard.edu/urn-3:HUL.InstRepos:20587664

\section{Terms of Use}

This article was downloaded from Harvard University's DASH repository, and is made available under the terms and conditions applicable to Other Posted Material, as set forth at http:// nrs.harvard.edu/urn-3:HUL.InstRepos:dash.current.terms-of-use\#LAA

\section{Share Your Story}

The Harvard community has made this article openly available.

Please share how this access benefits you. Submit a story.

\section{Accessibility}




\title{
THE ULTRAVIOLET-BRIGHT, SLOWLY DECLINING TRANSIENT PS1-11af AS A PARTIAL TIDAL DISRUPTION EVENT
}

\author{
R. Chornock ${ }^{1}$, E. Berger ${ }^{1}$, S. Gezari ${ }^{2}$, B. A. Zauderer ${ }^{1}$, A. Rest ${ }^{3}$, L. Chomiuk ${ }^{4}, 16$, A. Kamble ${ }^{1}$, A. M. Soderberg ${ }^{1}$, \\ I. Czekala ${ }^{1}$, J. Dittmann ${ }^{1}$, M. Drout ${ }^{1}$, R. J. Foley ${ }^{1,5}, 6,17$, W. Fong ${ }^{1}$, M. E. Huber ${ }^{7}$, R. P. Kirshner ${ }^{1}$, A. Lawrence ${ }^{8}$, \\ R. Lunnan ${ }^{1}$, G. H. Marion ${ }^{1,9}$, G. NARAyan ${ }^{1}$, A. G. Riess ${ }^{3,10}$, K. C. Roth ${ }^{11}$, N. E. Sanders ${ }^{1}$, D. Scolnic ${ }^{10}$, S. J. SmartT ${ }^{12}$, \\ K. Smith ${ }^{12}$, C. W. Stubbs ${ }^{1,13}$, J. L. Tonry ${ }^{7}$, W. S. Burgett ${ }^{7}$, K. C. Chambers ${ }^{7}$, H. Flewelling ${ }^{7}$, K. W. HodapP ${ }^{7}$, N. Kaiser ${ }^{7}$, \\ E. A. Magnier ${ }^{7}$, D. C. Martin ${ }^{14}$, J. D. Neill ${ }^{14}$, P. A. Price ${ }^{15}$, And R. Wainscoat ${ }^{7}$ \\ ${ }^{1}$ Harvard-Smithsonian Center for Astrophysics, 60 Garden St., Cambridge, MA 02138, USA; rchornock@cfa.harvard.edu \\ 2 Department of Astronomy, University of Maryland, College Park, MD 20742-2421, USA \\ ${ }^{3}$ Space Telescope Science Institute, 3700 San Martin Drive, Baltimore, MD 21218, USA \\ ${ }^{4}$ Department of Physics and Astronomy, Michigan State University, East Lansing, MI 48824, USA \\ ${ }^{5}$ Astronomy Department, University of Illinois at Urbana-Champaign, 1002 West Green Street, Urbana, IL 61801, USA \\ ${ }^{6}$ Department of Physics, University of Illinois at Urbana-Champaign, 1110 West Green Street, Urbana, IL 61801, USA \\ ${ }^{7}$ Institute for Astronomy, University of Hawaii, 2680 Woodlawn Drive, Honolulu, HI 96822, USA \\ ${ }^{8}$ Institute for Astronomy, University of Edinburgh Scottish Universities Physics Alliance, Royal Observatory, Blackford Hill, Edinburgh EH9 3HJ, UK \\ ${ }^{9}$ Department of Astronomy, University of Texas at Austin, Austin, TX 78712, USA \\ ${ }^{10}$ Department of Physics and Astronomy, Johns Hopkins University, 3400 North Charles Street, Baltimore, MD 21218, USA \\ ${ }^{11}$ Gemini Observatory, 670 North Aohoku Place, Hilo, HI 96720, USA \\ 12 Astrophysics Research Centre, School of Mathematics and Physics, Queen's University Belfast, Belfast, BT7 1NN, UK \\ ${ }^{13}$ Department of Physics, Harvard University, 17 Oxford Street, Cambridge, MA 02138, USA \\ ${ }^{14}$ Astronomy Department, California Institute of Technology, MC 249-17, 1200 East California Boulevard, Pasadena, CA 91125, USA \\ ${ }^{15}$ Department of Astrophysical Sciences, Princeton University, Princeton, NJ 08544, USA \\ Received 2013 September 11; accepted 2013 November 9; published 2013 December 11
}

\begin{abstract}
We present the Pan-STARRS1 discovery of the long-lived and blue transient PS1-11af, which was also detected by Galaxy Evolution Explorer with coordinated observations in the near-ultraviolet (NUV) band. PS1-11af is associated with the nucleus of an early type galaxy at redshift $z=0.4046$ that exhibits no evidence for star formation or active galactic nucleus activity. Four epochs of spectroscopy reveal a pair of transient broad absorption features in the UV on otherwise featureless spectra. Despite the superficial similarity of these features to P-Cygni absorptions of supernovae (SNe), we conclude that PS1-11af is not consistent with the properties of known types of SNe. Blackbody fits to the spectral energy distribution are inconsistent with the cooling, expanding ejecta of a $\mathrm{SN}$, and the velocities of the absorption features are too high to represent material in homologous expansion near a SN photosphere. However, the constant blue colors and slow evolution of the luminosity are similar to previous optically selected tidal disruption events (TDEs). The shape of the optical light curve is consistent with models for TDEs, but the minimum accreted mass necessary to power the observed luminosity is only $\sim 0.002 M_{\odot}$, which points to a partial disruption model. A full disruption model predicts higher bolometric luminosities, which would require most of the radiation to be emitted in a separate component at high energies where we lack observations. In addition, the observed temperature is lower than that predicted by pure accretion disk models for TDEs and requires reprocessing to a constant, lower temperature. Three deep non-detections in the radio with the Very Large Array over the first two years after the event set strict limits on the production of any relativistic outflow comparable to Swift J1644+57, even if off-axis.
\end{abstract}

Key words: accretion, accretion disks - black hole physics - galaxies: nuclei

Online-only material: color figures

\section{INTRODUCTION}

When a star of mass $M_{\star}$ and radius $R_{\star}$ has an orbit with a pericenter passage sufficiently close to a black hole of mass $M_{\mathrm{bh}}$, as in the nucleus of a galaxy, it can be torn apart by tidal forces (Hills 1975). Approximately half of the debris of the star becomes unbound and leaves the system on hyperbolic orbits, while the other half remains bound to the black hole on elliptical orbits. When the bound material returns to pericenter, it can then accrete onto the black hole and produce an optical transient (Rees 1988), called a tidal disruption event (TDE). TDEs are signatures of the presence of otherwise quiescent black holes

\footnotetext{
${ }^{16}$ Jansky Fellow.

${ }^{17}$ Clay Fellow.
}

and, with better understanding of the relevant physics, may prove useful in the long run both as probes of black hole masses in distant galaxies and for the study of accretion processes in a different regime than that of active galactic nuclei (AGNs).

The condition for disruption is that the pericenter distance of the orbit is less than the tidal radius, $r_{t}=R_{\star}\left(M_{\mathrm{bh}} / M_{\star}\right)^{1 / 3}$. For solar-type stars disrupted by $10^{6} M_{\odot}$ black holes, the characteristic blackbody $(\mathrm{BB})$ temperature $\left(T_{\mathrm{BB}}\right)$ for a black hole accreting at the Eddington rate at this radius is $\sim 2.5 \times 10^{5} \mathrm{~K}$ (e.g., Ulmer 1999; Strubbe \& Quataert 2009). The radiation output is therefore expected to peak in the extreme-ultraviolet (UV) and X-ray bands. The characteristic light curve behavior prediction for TDEs involves a rapid rise to maximum light, with a decline after the peak that falls as $t^{-5 / 3}$ (Rees 1988; Evans \& Kochanek 1989). 
However, more detailed modeling has shown that actual TDEs should exhibit more complex behavior. The derivation of the $t^{-5 / 3}$ light curve relies on the assumption that the spread of specific energy with mass for the stellar debris is constant, but recent work has shown that the internal structure of the star can modify these expectations (Ramirez-Ruiz \& Rosswog 2009; Lodato et al. 2009; Stone et al. 2013; Guillochon \& RamirezRuiz 2013), with faster decline rates predicted immediately after the peak. Furthermore, most studies have concentrated on complete stellar disruptions at the tidal radius, while real disruptions can occur at a range of pericenter distances and partial disruptions outside of the nominal tidal radius may also contribute to the flare rate (Guillochon \& Ramirez-Ruiz 2013). Finally, the conversion of accreting mass, $\dot{M}_{\text {acc }}$, to observable radiation is not a simple process. Models of the spectral energy distributions (SEDs) of TDEs and their evolution have grown increasingly complex over time, starting with thin disk models and adding thick disks and outflows or winds to model superEddington accretion (Loeb \& Ulmer 1997; Ulmer 1999; Strubbe \& Quataert 2009, 2011; Lodato \& Rossi 2011; Guillochon et al. 2013).

Two relativistic TDEs, Swift J164449.3+573451 (Sw $1644+57$; Bloom et al. 2011; Levan et al. 2011; Burrows et al. 2011; Zauderer et al. 2011) and Swift J2058.4+0516 (Sw $2058+05$; Cenko et al. 2012b) have been discovered on the basis of $\gamma$-ray triggers. These objects have $\mathrm{X}$-ray light curves that approximately match the $t^{-5 / 3}$ decline rate, and appear to have launched relativistic jets along the line of sight (Giannios \& Metzger 2011), adding another potential emission component to the SED.

Most of the TDE candidates reported in the literature are large amplitude soft X-ray flares from galaxy nuclei (e.g., Bade et al. 1996; Komossa \& Bade 1999; Li et al. 2002; Komossa et al. 2004; Esquej et al. 2008; Maksym et al. 2013). These generally have poorly sampled light curves, but have the predicted soft spectra and light curve decay rates that are consistent with a $t^{-5 / 3}$ decline, for suitable assumptions about the time of disruption. Gezari et al. (2009b) presented Galaxy Evolution Explorer (GALEX) observations of three TDE candidates with SEDs that had $T_{\mathrm{BB}} \gtrsim 5 \times 10^{4} \mathrm{~K}$ and light curves exhibiting evidence of $t^{-5 / 3}$ declines. Cenko et al. (2012a) interpreted the fast fading and luminous nuclear transient PTF 10iya as the early super-Eddington phase of accretion in a TDE.

van Velzen et al. (2011a) discovered the first two optically selected TDE candidates in repeated Sloan Digital Sky Survey (SDSS) imaging of Stripe 82, which we denote SDSS TDE1 and SDSS TDE2. These transients were selected on the basis of their unusually blue colors and slow evolution, which made them stand out from normal supernovae (SNe) and AGN variability. Subsequently, Gezari et al. (2012) described the Pan-STARRS1 (PS1) discovery of PS1-10jh, the first TDE with a well-sampled optical light curve on both the rise and decline from maximum light, which makes it a benchmark object for studies of the TDE process (e.g., Guillochon et al. 2013; Bogdanovic et al. 2013). The spectra of SDSS TDE2 exhibited broad $\mathrm{H} \alpha$ emission, while PS1-10jh had broad He II emission lines. These were the first definitive detections of spectral features from TDEs, although some galaxies in the literature with unusual nuclear spectra have been claimed to be produced by the late-time effects of TDEs (Storchi-Bergmann et al. 1995; Bogdanović et al. 2004; Komossa et al. 2008; Wang et al. 2012; Yang et al. 2013).

In this work, we describe the discovery of a new TDE by the PS1 survey, PS1-11af, which is the first TDE to exhibit broad UV absorption features. We give a description of the optical, UV, and radio observations in Section 2. In Section 3, we analyze the host galaxy and set upper limits on star formation and AGN activity. We describe the evolution of the SED and isolate the spectrum of the transient in Section 4. In Section 5, we examine a SN interpretation for the properties of PS1-11af and find that it cannot be fit by any known model. We then interpret PS1-11af as a TDE in Section 6 before concluding in Section 7. Throughout this paper, we adopt the flat $\Lambda \mathrm{CDM}$ Planck+WMAP+high- $\ell+\mathrm{BAO}$ cosmology of Ade et al. (2013) with $H_{0}=68 \mathrm{~km} \mathrm{~s}^{-1} \mathrm{Mpc}^{-1}, \Omega_{\mathrm{M}}=0.31$, and $\Omega_{\Lambda}=0.69$. All quoted dates are UT, and all magnitudes are reported on the AB scale.

\section{OBSERVATIONS}

\subsection{Discovery and Photometry}

The PS1 telescope has a $1.8 \mathrm{~m}$ diameter primary mirror that images a field with a diameter of 3.3 (Hodapp et al. 2004) onto a total of $604800 \times 4800$ pixel detectors, with a pixel scale of 0.258 (Tonry \& Onaka 2009). A more complete description of the PS1 system, hardware and software, is provided by Kaiser et al. (2010). The nightly PS1 Medium Deep Survey (MDS) observations are obtained through a set of five broadband filters, designated as $g_{\mathrm{P} 1}, r_{\mathrm{P} 1}, i_{\mathrm{P} 1}, z_{\mathrm{P} 1}$, and $y_{\mathrm{P} 1}$, with a typical cadence of $3 \mathrm{~d}$ between observations in $g_{\mathrm{P} 1} r_{\mathrm{P} 1} i_{\mathrm{P} 1} z_{\mathrm{P} 1}$. Although the filter system for PS1 has much in common with that used in previous surveys, such as the SDSS (Ahn et al. 2012), there are differences, with further information on the passband shapes described by Stubbs et al. (2010). Photometry is reported in the "natural" PS1 system, $m=-2.5 \log \left(f_{v}\right)+m^{\prime}$, with a single zeropoint adjustment $m^{\prime}$ made in each band to conform to the AB magnitude scale (Tonry et al. 2012). PS1 magnitudes are interpreted as being at the top of the atmosphere, with 1.2 airmasses of atmospheric attenuation being included in the system response function.

PS1 data are processed through the Image Processing Pipeline (IPP; Magnier 2006) on a computer cluster at the Maui High Performance Computer Center. The pipeline runs the images through a succession of stages, including flat-fielding ("detrending"), a flux-conserving warping to a sky-based image plane, masking and artifact removal, and object detection and photometry. Transient detection using IPP photometry is carried out at Queen's University Belfast. Independently, difference images are produced from the stacked nightly MDS images by the photpipe pipeline (Rest et al. 2005) running on the Odyssey computer cluster at Harvard University. The discovery and data presented here are from the photpipe analysis.

We first detected PS1-11af in $g_{\mathrm{P} 1} r_{\mathrm{P} 1}$ images obtained on the night of 2010 December 30 after non-detections in our first observations of that field for the observing season on 2010 December $15 / 16\left(g_{\mathrm{P} 1} r_{\mathrm{P} 1} i_{\mathrm{P} 1}\right)$. The transient rose to a peak in late January of 2011 and slowly faded thereafter, remaining detectable in PS1 imaging when observations ceased at the end of April due to solar conjunction. The mean position of PS1-11af was $\alpha=9^{\mathrm{h}} 57^{\mathrm{m}} 26^{\mathrm{s}} .815, \delta=+03^{\circ} 14^{\prime} 01^{\prime \prime}$.00 (J2000), with an uncertainty of $0^{\prime \prime} .1$ in each coordinate. False-color images of PS1-11af near maximum light and its host galaxy are shown in Figure 1. The blue color of the transient relative to its red host galaxy is immediately apparent.

We construct deep template observations from the preoutburst images and subtract them from the PS1 observations 

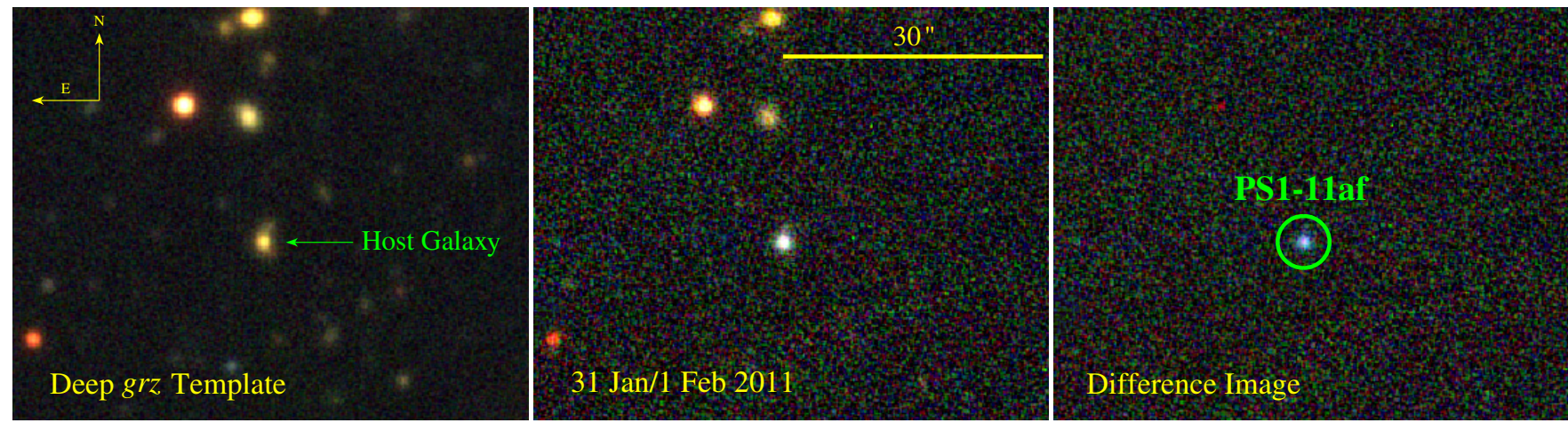

Figure 1. False-color images of the field of PS1-11af, with $g_{\mathrm{P} 1} r_{\mathrm{P} 1} z_{\mathrm{P} 1}$ mapped to blue, green, and red, respectively. The logarithmic color scales are the same in each panel. The left panel displays the deep template images created from pre-outburst PS1 data. The image in the middle panel was formed from images taken on consecutive nights shortly after peak of the outburst. The right panel is the difference image formed by subtraction of the template images on the left from the images in the middle. Note the strong blue color of the transient relative to its host galaxy and other field sources.

(A color version of this figure is available in the online journal.)

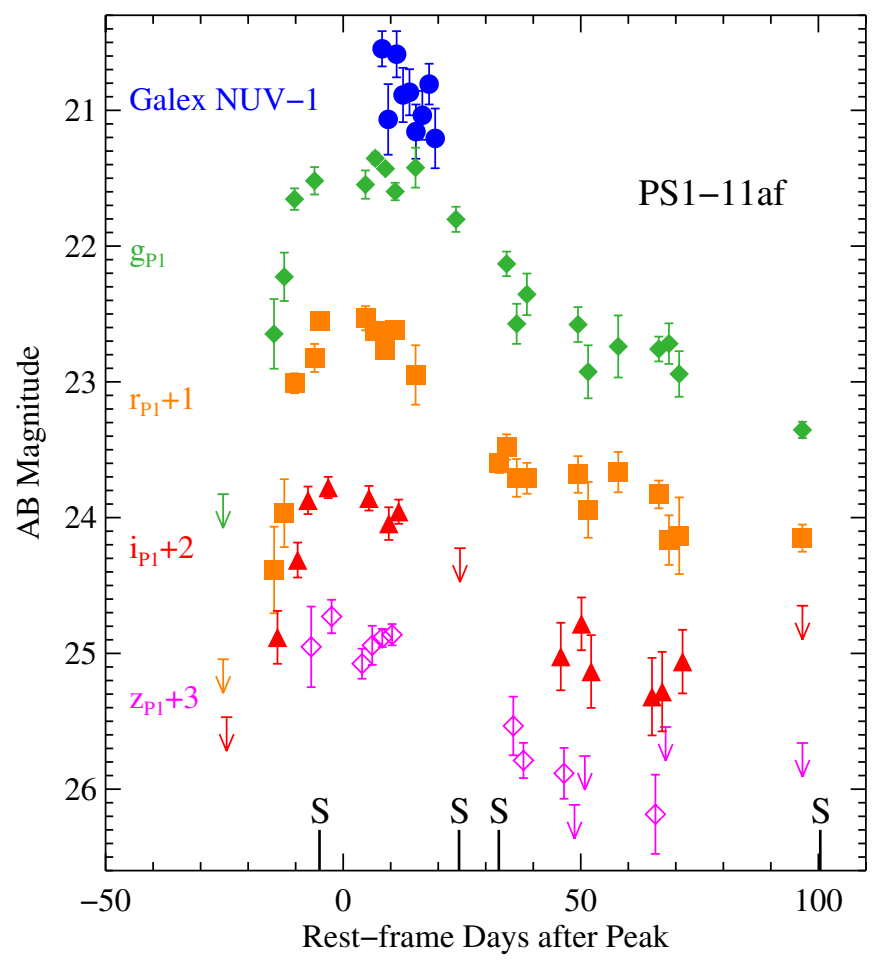

Figure 2. Light curve of PS1-11af. The blue circles are from the GALEX NUV band, with the $g_{\mathrm{P} 1} r_{\mathrm{P} 1} i_{\mathrm{P} 1} z_{\mathrm{P} 1}$ photometry below, and the constant offsets are noted. Vertical lines marked with an "S" show the dates of the spectroscopic observations.

(A color version of this figure is available in the online journal.)

using photpipe (Rest et al. 2005). Details of the photometry and generation of PS1 transient light curves are given by Rest et al. (2013) and Scolnic et al. (2013). The final PS1-11af photometry, after correction for $E(B-V)=0.027$ mag of Galactic extinction (Schlafly \& Finkbeiner 2011; Schlegel et al. 1998), is given in Table 1 and shown in Figure 2. Fourth-order polynomial fits to the $g_{\mathrm{P} 1}$ and $r_{\mathrm{P} 1}$ light curves near peak give dates for maximum light at Modified Julian Dates (MJDs) of $55581 \pm 2$ days (=2011 January 20), which we adopt as the time of peak throughout this paper.

The field of PS1-11af was monitored in the near-ultraviolet band (NUV; Morrissey et al. 2007) as part of the GALEX Time Domain Survey (TDS; Gezari et al. 2013) from 2011
January 31 to 2011 February 16 . The transient was detected in each observation during this period, while nothing was detected at the position of PS1-11 af in either of the NUV or far-ultraviolet (FUV) bands in prior GALEX observations in 2008 and 2010. We perform aperture photometry of PS1-11af during outburst and list the results in Table 1. Further details of the GALEX survey design and data products were presented by Gezari et al. (2013).

In addition, we observed the host of PS1-11af on 2013 May 20 in $J$ and $H$ for a total of 1200 and 1254 s, respectively, using the FourStar Infrared Camera (Persson et al. 2008) on the $6.5 \mathrm{~m}$ Magellan Baade telescope. The images were flat fielded, sky subtracted, and stacked with standard tasks in $\operatorname{IRAF}^{18}$ using the instrument pipeline. The photometry was calibrated using 2MASS stars in the field.

\subsection{Spectroscopy}

We obtained four epochs of spectroscopy of PS1-11af in outburst between 2011 January 13 and 2011 June 10 using the Low Dispersion Survey Spectrograph-3 (LDSS3; an updated version of LDSS-2, Allington-Smith et al. 1994) on the $6.5 \mathrm{~m}$ Magellan Clay telescope, the Blue Channel Spectrograph (BC; Schmidt et al. 1989) on the $6.5 \mathrm{~m}$ MMT, the Inamori-Magellan Areal Camera and Spectrograph (IMACS; Dressler et al. 2006) on the Magellan Baade telescope, and the Gemini Multi-Object Spectrograph (GMOS; Hook et al. 2004) on the $8 \mathrm{~m}$ GeminiSouth telescope. A full log with details of the observations is given in Table 2. Conditions were clear for the initial LDSS3 and $\mathrm{BC}$ observations, but poor and variable seeing compromised the IMACS spectra. We combine the IMACS spectra on consecutive nights to increase the signal-to-noise ratio $(\mathrm{S} / \mathrm{N})$.

After PS1-11af had faded away, we returned to obtain spectroscopy of the host galaxy using similar BC and LDSS3 setups as those used for the original observations, including the same slit position angles (PAs) to within $5^{\circ}$. We also obtained spectroscopy with LDSS3 using a significantly redder setup, designed to cover $\mathrm{H} \alpha$ at the rest frame of the host galaxy. A few additional host spectra were obtained at $\mathrm{PA}=-10^{\circ}$ for reasons discussed below but only when that angle was close to the parallactic angle.

\footnotetext{
18 IRAF is distributed by the National Optical Astronomy Observatory, which is operated by the Association of Universities for Research in Astronomy, Inc., under cooperative agreement with the National Science Foundation.
} 
Table 1

PS1-11af Photometry

\begin{tabular}{|c|c|c|c|c|c|}
\hline MJD & $\begin{array}{l}\text { Epoch }^{\mathrm{a}} \\
\text { (d) }\end{array}$ & Filter & $\begin{array}{c}\text { Magnitude }^{b} \\
\text { (AB) }\end{array}$ & Error & Instrument \\
\hline 55592.5 & 8.2 & NUV & 21.55 & 0.13 & $G A L E X$ \\
\hline 55594.3 & 9.5 & NUV & 22.07 & 0.26 & $G A L E X$ \\
\hline 55596.8 & 11.2 & NUV & 21.59 & 0.17 & $G A L E X$ \\
\hline 55598.7 & 12.6 & NUV & 21.89 & 0.20 & $G A L E X$ \\
\hline 55600.6 & 14.0 & NUV & 21.87 & 0.17 & $G A L E X$ \\
\hline 55602.5 & 15.3 & NUV & 22.16 & 0.20 & $G A L E X$ \\
\hline 55604.4 & 16.7 & NUV & 22.04 & 0.18 & $G A L E X$ \\
\hline 55606.4 & 18.1 & NUV & 21.81 & 0.15 & $G A L E X$ \\
\hline 55608.2 & 19.4 & NUV & 22.21 & 0.22 & $G A L E X$ \\
\hline 55545.5 & -25.3 & $g_{\mathrm{P} 1}$ & $>23.83$ & $\ldots$ & PS1 \\
\hline 55560.6 & -14.5 & $g_{\mathrm{P} 1}$ & 22.65 & 0.26 & PS1 \\
\hline 55563.6 & -12.4 & $g_{\mathrm{P} 1}$ & 22.23 & 0.18 & PS1 \\
\hline 55566.6 & -10.3 & $g_{\mathrm{P} 1}$ & 21.65 & 0.08 & PS1 \\
\hline 55572.5 & -6.1 & $g_{\mathrm{P} 1}$ & 21.52 & 0.10 & PS1 \\
\hline 55587.6 & 4.7 & $g_{\mathrm{P} 1}$ & 21.55 & 0.10 & PS1 \\
\hline 55590.5 & 6.8 & $g_{\mathrm{P} 1}$ & 21.35 & 0.04 & PS1 \\
\hline 55593.5 & 8.9 & $g_{\mathrm{P} 1}$ & 21.43 & 0.03 & PS1 \\
\hline 55596.4 & 11.0 & $g_{\mathrm{P} 1}$ & 21.60 & 0.06 & PS1 \\
\hline 55602.4 & 15.2 & $g_{\mathrm{P} 1}$ & 21.42 & 0.15 & PS1 \\
\hline 55614.3 & 23.7 & $g_{\mathrm{P} 1}$ & 21.80 & 0.09 & PS1 \\
\hline 55629.3 & 34.4 & $g_{\mathrm{P} 1}$ & 22.13 & 0.09 & PS1 \\
\hline 55632.3 & 36.5 & $g_{\mathrm{P} 1}$ & 22.57 & 0.15 & PS1 \\
\hline 55635.3 & 38.7 & $g_{\mathrm{P} 1}$ & 22.36 & 0.15 & PS1 \\
\hline 55650.4 & 49.4 & $g_{\mathrm{P} 1}$ & 22.58 & 0.13 & PS1 \\
\hline 55653.4 & 51.5 & $g_{\mathrm{P} 1}$ & 22.93 & 0.20 & PS1 \\
\hline 55662.3 & 57.9 & $g_{\mathrm{P} 1}$ & 22.74 & 0.23 & PS1 \\
\hline 55674.3 & 66.4 & $g_{\mathrm{P} 1}$ & 22.76 & 0.09 & PS1 \\
\hline 55677.3 & 68.6 & $g_{\mathrm{P} 1}$ & 22.72 & 0.15 & PS1 \\
\hline 55680.3 & 70.7 & $g_{\mathrm{P} 1}$ & 22.94 & 0.17 & PS1 \\
\hline 55716.8 & 96.7 & $g^{\prime}$ & 23.35 & 0.06 & GMOS \\
\hline 55545.5 & -25.3 & $r_{\mathrm{P} 1}$ & $>24.04$ & $\cdots$ & PS1 \\
\hline 55560.6 & -14.5 & $r_{\mathrm{P} 1}$ & 23.39 & 0.32 & PS1 \\
\hline 55563.6 & -12.4 & $r_{\mathrm{P} 1}$ & 22.97 & 0.25 & PS1 \\
\hline 55566.6 & -10.3 & $r_{\mathrm{P} 1}$ & 22.01 & 0.07 & PS1 \\
\hline 55572.6 & -6.0 & $r_{\mathrm{P} 1}$ & 21.82 & 0.10 & PS1 \\
\hline 55574.3 & -4.8 & $r^{\prime}$ & 21.55 & 0.03 & LDSS3 \\
\hline 55587.6 & 4.7 & $r_{\mathrm{P} 1}$ & 21.53 & 0.09 & PS1 \\
\hline 55590.5 & 6.8 & $r_{\mathrm{P} 1}$ & 21.62 & 0.05 & PS1 \\
\hline 55593.5 & 8.9 & $r_{\mathrm{P} 1}$ & 21.77 & 0.05 & PS1 \\
\hline 55596.4 & 11.0 & $r_{\mathrm{P} 1}$ & 21.62 & 0.06 & PS1 \\
\hline 55602.4 & 15.2 & $r_{\mathrm{P} 1}$ & 21.95 & 0.22 & PS1 \\
\hline 55627.1 & 32.8 & $r^{\prime}$ & 22.60 & 0.07 & IMACS \\
\hline 55629.3 & 34.4 & $r_{\mathrm{P} 1}$ & 22.48 & 0.09 & PS1 \\
\hline 55632.3 & 36.5 & $r_{\mathrm{P} 1}$ & 22.71 & 0.14 & PS1 \\
\hline 55635.3 & 38.7 & $r_{\mathrm{P} 1}$ & 22.71 & 0.11 & PS1 \\
\hline 55650.4 & 49.4 & $r_{\mathrm{P} 1}$ & 22.68 & 0.13 & PS1 \\
\hline 55653.4 & 51.5 & $r_{\mathrm{P} 1}$ & 22.94 & 0.21 & PS1 \\
\hline 55662.3 & 57.9 & $r_{\mathrm{P} 1}$ & 22.66 & 0.15 & PS1 \\
\hline 55674.3 & 66.4 & $r_{\mathrm{P} 1}$ & 22.83 & 0.10 & PS1 \\
\hline 55677.3 & 68.6 & $r_{\mathrm{P} 1}$ & 23.17 & 0.18 & PS1 \\
\hline 55680.3 & 70.7 & $r_{\mathrm{P} 1}$ & 23.13 & 0.28 & PS1 \\
\hline 55716.8 & 96.7 & $r^{\prime}$ & 23.15 & 0.10 & GMOS \\
\hline 55546.5 & -24.6 & $i_{\mathrm{P} 1}$ & $>23.47$ & $\ldots$ & PS1 \\
\hline 55561.6 & -13.8 & $i_{\mathrm{P} 1}$ & 22.88 & 0.19 & PS1 \\
\hline 55567.5 & -9.6 & $i_{\mathrm{P} 1}$ & 22.31 & 0.13 & PS1 \\
\hline 55570.6 & -7.4 & $i_{\mathrm{P} 1}$ & 21.87 & 0.10 & PS1 \\
\hline 55576.6 & -3.1 & $i_{\mathrm{P} 1}$ & 21.78 & 0.08 & PS1 \\
\hline 55588.6 & 5.4 & $i_{\mathrm{P} 1}$ & 21.86 & 0.09 & PS1 \\
\hline 55594.5 & 9.6 & $i_{\mathrm{P} 1}$ & 22.04 & 0.12 & PS1 \\
\hline 55597.4 & 11.7 & $i_{\mathrm{P} 1}$ & 21.96 & 0.09 & PS1 \\
\hline 55615.5 & 24.6 & $i_{\mathrm{P} 1}$ & $>22.23$ & $\cdots$ & PS1 \\
\hline 55645.3 & 45.8 & $i_{\mathrm{P} 1}$ & 23.02 & 0.25 & PS1 \\
\hline 55651.4 & 50.1 & $i_{\mathrm{P} 1}$ & 22.78 & 0.19 & PS1 \\
\hline 55654.3 & 52.2 & $i_{\mathrm{P} 1}$ & 23.13 & 0.27 & PS1 \\
\hline
\end{tabular}

Table 1

(Continued)

\begin{tabular}{|c|c|c|c|c|c|}
\hline MJD & $\begin{array}{l}\text { Epoch }^{\mathrm{a}} \\
\text { (d) }\end{array}$ & Filter & $\begin{array}{c}\text { Magnitude } \\
\text { (AB) }\end{array}$ & Error & Instrument \\
\hline 55672.3 & 65.0 & $i_{\mathrm{P} 1}$ & 23.32 & 0.29 & PS1 \\
\hline 55675.3 & 67.1 & $i_{\mathrm{P} 1}$ & 23.28 & 0.29 & PS1 \\
\hline 55681.3 & 71.4 & $i_{\mathrm{P} 1}$ & 23.06 & 0.23 & PS1 \\
\hline 55716.8 & 96.7 & $i^{\prime}$ & $>22.65$ & $\cdots$ & GMOS \\
\hline 55571.6 & -6.7 & $z_{\mathrm{P} 1}$ & 21.95 & 0.30 & PS1 \\
\hline 55577.6 & -2.4 & $z_{\mathrm{P} 1}$ & 21.73 & 0.12 & PS1 \\
\hline 55586.6 & 4.0 & $z_{\mathrm{P} 1}$ & 22.08 & 0.11 & PS1 \\
\hline 55589.6 & 6.1 & $z_{\mathrm{P} 1}$ & 21.94 & 0.14 & PS1 \\
\hline 55592.6 & 8.3 & $z_{\mathrm{P} 1}$ & 21.89 & 0.07 & PS1 \\
\hline 55595.5 & 10.3 & $z_{\mathrm{P} 1}$ & 21.86 & 0.08 & PS1 \\
\hline 55631.3 & 35.8 & $z_{\mathrm{P} 1}$ & 22.53 & 0.22 & PS1 \\
\hline 55634.3 & 37.9 & $z_{\mathrm{P} 1}$ & 22.79 & 0.13 & PS1 \\
\hline 55646.3 & 46.5 & $z_{\mathrm{P} 1}$ & 22.88 & 0.19 & PS1 \\
\hline 55649.4 & 48.7 & $z_{\mathrm{P} 1}$ & $>23.12$ & $\ldots$ & PS1 \\
\hline 55652.4 & 50.8 & $z_{\mathrm{P} 1}$ & $>22.76$ & $\ldots$ & PS1 \\
\hline 55673.3 & 65.7 & $z_{\mathrm{P} 1}$ & 23.18 & 0.29 & PS1 \\
\hline 55676.3 & 67.8 & $z_{\mathrm{P} 1}$ & $>22.54$ & $\cdots$ & PS1 \\
\hline 55716.8 & 96.7 & $z^{\prime}$ & $>22.66$ & $\cdots$ & GMOS \\
\hline 55641.5 & 43.1 & $y_{\mathrm{P} 1}$ & 21.77 & 0.49 & PS1 \\
\hline 55668.4 & 62.2 & $y_{\mathrm{P} 1}$ & $>21.24$ & $\cdots$ & PS1 \\
\hline 55669.3 & 62.9 & $y_{\mathrm{P} 1}$ & $>21.46$ & $\cdots$ & PS1 \\
\hline 55671.3 & 64.3 & $y_{\mathrm{P} 1}$ & $>21.28$ & $\ldots$ & PS1 \\
\hline
\end{tabular}

Notes.

${ }^{\text {a }}$ In rest-frame days relative to maximum light on MJD 55581.0.

${ }^{\mathrm{b}}$ Corrected for Galactic reddening. Upper limits are $3 \sigma$.

We also obtained several images as part of the acquisition process using LDSS3, IMACS, and GMOS. We process the two-dimensional frames using standard tasks in IRAF. Multiple attempts to obtain the GMOS spectrum over the course of several days were aborted due to bad weather so we only obtained a $g^{\prime} r^{\prime} i^{\prime} z^{\prime}$ acquisition sequence on each date. We stack the images in each filter and report the average dates in Table 1. We subtract the PS1 templates in the appropriate filters from the acquisition images and calibrate the photometry to PS1 stars in the field.

We reduce the spectroscopic data using standard tasks in IRAF along with our own IDL procedures to apply a flux calibration and correct for telluric absorption. The longslits were always aligned within $10^{\circ}$ of the parallactic angle to mitigate the possible effects of differential atmospheric dispersion (Filippenko 1982). We did not use order-blocking filters, so second-order light contamination is a concern with a source this blue. Our early LDSS3 observation was taken using the standard 0.75 longslit located $2^{\prime}$ blueward of the center of the field combined with the VPH-all grism. Past experience, along with tests using order-blocking filters on blue standard stars, has demonstrated that this instrument setup combination suffers very little contamination from second-order light. More generally, we use observations of both relatively blue (sdO spectral types) and red (F-type) standard stars taken both with and without order-blocking filters to define the flux calibrations for the $\mathrm{BC}$ and Magellan spectra. Despite our best efforts, we caution that some contamination may still be present at observed wavelengths longward of $\sim 8000 \AA$. For GMOS-S, we use archival observations of EG21 to define the flux calibration. The final spectra are presented in Figure 3. All spectra exhibit absorption from the $\mathrm{Ca}$ II $\mathrm{H}+\mathrm{K}$ doublet at redshift $z=0.405$, which we refine in Section 3.1 to $z=0.4046$ and adopt as the host redshift throughout this paper. 
Table 2

Log of Spectroscopic Observations

\begin{tabular}{|c|c|c|c|c|c|c|c|c|c|}
\hline $\begin{array}{l}\text { UT Midpoint } \\
\text { (YYYY-MM-DD.DD) }\end{array}$ & $\begin{array}{l}\text { Epoch }^{\mathrm{a}} \\
\text { (days) }\end{array}$ & Instrument & $\begin{array}{l}\text { Wavelength } \\
\text { Range }(\AA)\end{array}$ & $\begin{array}{l}\text { Slit } \\
\left({ }^{\prime \prime}\right)\end{array}$ & $\begin{array}{l}\text { Grating/ } \\
\text { Grism }\end{array}$ & Filter & $\begin{array}{l}\text { Exposure } \\
\text { Time (s) }\end{array}$ & $\begin{array}{c}\text { Mean } \\
\text { Airmass }\end{array}$ & $\begin{array}{c}\text { Position } \\
\text { Angle }\left(^{\circ}\right)\end{array}$ \\
\hline $2011-01-13.27$ & -5 & LDSS3 & $3650-9460$ & 0.75 & VPH-all & none & 1500 & 1.20 & 20 \\
\hline 2011-03-06.18 & 32 & IMACS & $4000-10100$ & 0.9 & $300 /+17.5$ & none & 2400 & 1.20 & 167 \\
\hline 2011-03-07.17 & 33 & IMACS & $4000-10100$ & 0.9 & $300 /+17.5$ & none & 3600 & 1.18 & 177 \\
\hline 2011-06-10.02 & 100 & GMOS-S & $3500-6280$ & 1.0 & B600 & none & 3600 & 1.77 & 315 \\
\hline \multicolumn{10}{|c|}{ Host Spectra } \\
\hline $2011-12-29.39$ & 244 & Blue Channel & $3320-8550$ & 1.0 & 300 & none & 5400 & 1.22 & 135 \\
\hline $2013-01-11 / 14^{b}$ & 515 & LDSS3 & $3650-9450$ & 0.75 & VPH-all & none & 2900 & 1.22 & $22 /-10$ \\
\hline $2013-01-12 / 13^{b}$ & 515 & LDSS3 & $5970-9300$ & 1.0 & VPH-red & OG590 & 5700 & 1.20 & $17 /-10$ \\
\hline
\end{tabular}

Notes.

a In rest-frame days relative to maximum light.

b Spectra taken on two nights.

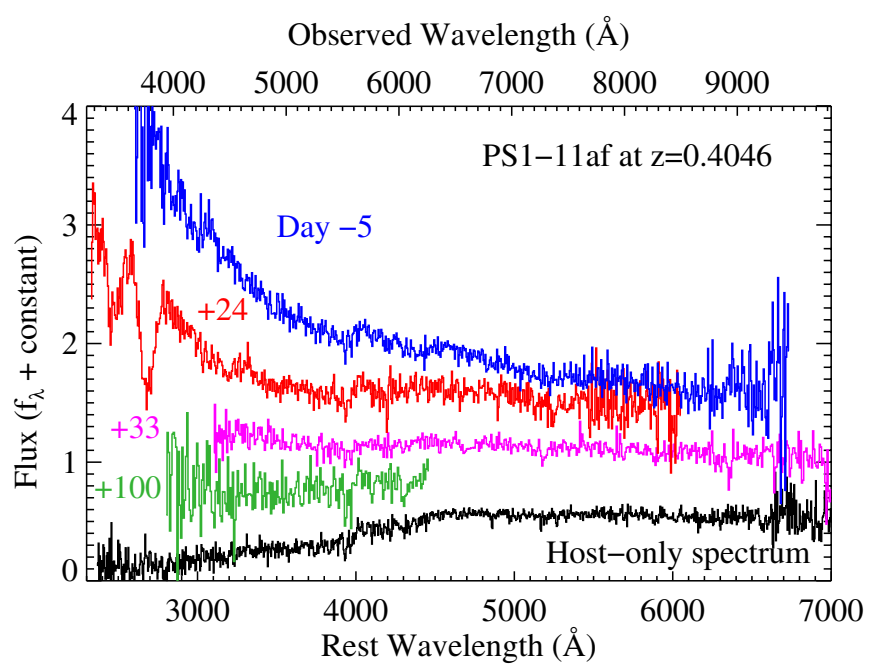

Figure 3. Observed spectra of PS1-11af with constant flux offsets for clarity. The spectra are labeled with the number of rest-frame days relative to peak. Note the blue colors at early times and the two deep UV absorption features on Day +24 . The bottom spectrum (black) shows the combined spectrum of the host galaxy after the transient faded.

(A color version of this figure is available in the online journal.)

\subsection{Radio Observations}

We observed the field of PS1-11af with the Karl G. Jansky Very Large Array (VLA; Dougherty \& Perley 2010) three times. The first epoch was 2011 March 29.03 (Project 10A-214; PI: Soderberg), and subsequent epochs were obtained beginning 2012 January 7.38 (Project 11B-192; PI: Chomiuk) and 2013 May 31.96 (Project 13A-437; PI: Soderberg). Our observations are summarized in Table 3. The first observation was conducted with the old VLA system with two $128 \mathrm{MHz}$ windows centered at 4.8 and $5.0 \mathrm{GHz}$. The latter two observations were conducted with the new WIDAR correlator (Perley et al. 2011) at a mean frequency of $5.9 \mathrm{GHz}$ (lower sideband frequency centered at $5.0 \mathrm{GHz}$; upper sideband frequency centered at $6.75 \mathrm{GHz}$ ).

In all epochs, we use standard data reduction procedures in AIPS (Greisen 2003). We use 3C286 for bandpass and flux calibration, and J1024-0052 for gain calibration. We flag and excise channels affected by radio frequency interference, which results in an effective bandwidth of $\sim 1.7 \mathrm{GHz}$. A $\sim 35 \mathrm{mJy}$ source $4^{\prime}$ from the position of PS1-11af is used for selfcalibration. We do not detect significant radio emission from
Table 3

PS1-11af VLA Observations

\begin{tabular}{lcccc}
\hline \hline MJD & $\begin{array}{c}\text { Epoch }^{\mathrm{a}} \\
(\mathrm{d})\end{array}$ & $\begin{array}{c}\text { On-source } \\
\text { Time }(\mathrm{min})\end{array}$ & $\begin{array}{c}\text { Frequency } \\
(\mathrm{GHz})\end{array}$ & $\begin{array}{c}3 \sigma \text { Upper } \\
\text { Limit }(\mu \mathrm{Jy})\end{array}$ \\
\hline 55649.0 & 48 & 36 & 4.9 & $<51$ \\
55933.4 & 251 & 17 & 5.5 & $<30$ \\
56444.0 & 614 & 60 & 5.5 & $<45$ \\
\hline
\end{tabular}

Note. ${ }^{a}$ In rest-frame days relative to maximum light.

PS1-11af in any epoch and set $3 \sigma$ limits of 51,30 , and $45 \mu \mathrm{Jy}$, respectively, from the root-mean-squared (rms) flux values of the images.

\section{HOST GALAXY}

The host galaxy of PS1-11af is shown in the left panel of Figure 1. It is apparent that the host is relatively red and the core is rather symmetric, indicative of a bulge-dominated or early type galaxy. The host is marginally resolved in the PS1 template images (seeing FWHM $\approx 1^{\prime \prime}$. 1 ). An asymmetric extension of flux to the north-northwest $\left(\mathrm{PA} \approx-10^{\circ}\right)$ is visible. In our images obtained in the best seeing (with LDSS3 in $g^{\prime}$ and FourStar), this extension appears somewhat offset from the main part of the galaxy. The offset $\left(\sim 2^{\prime \prime}\right)$ is $11 \mathrm{kpc}$ at this redshift. It is not immediately apparent whether this represents substructure within the host galaxy or possibly a companion galaxy.

We use the PS1 image with our highest-significance detection of PS1-11af in $r_{\mathrm{P} 1}$ (MJD $\left.=55590.5\right)$ to perform relative astrometry between the deep template image and the detection of the source. Forty common point sources were used to tie the two images together, with a rms dispersion of 28 mas in each coordinate. The offset between the detection of PS1-11af and the core of its host galaxy in the template is $60 \pm 62$ mas, including the uncertainties in the object and nuclear centroids, consistent with PS1-11af being a nuclear event. However, the $3 \sigma$ uncertainty corresponds to $1 \mathrm{kpc}$, which contains a significant fraction of the stars in this compact host galaxy.

We collect photometry of the host galaxy of PS1-11af using the deep PS1 template images and FourStar data described in Section 2.1. Because of the ambiguous origin of the flux extension, we report aperture photometry in Table 4 using apertures of radius 1 1. 15 and 3". 0 . The narrower aperture captures the main core of the galaxy (possibly the bulge), while the wider one includes the extension plus the wings of the central galaxy. 


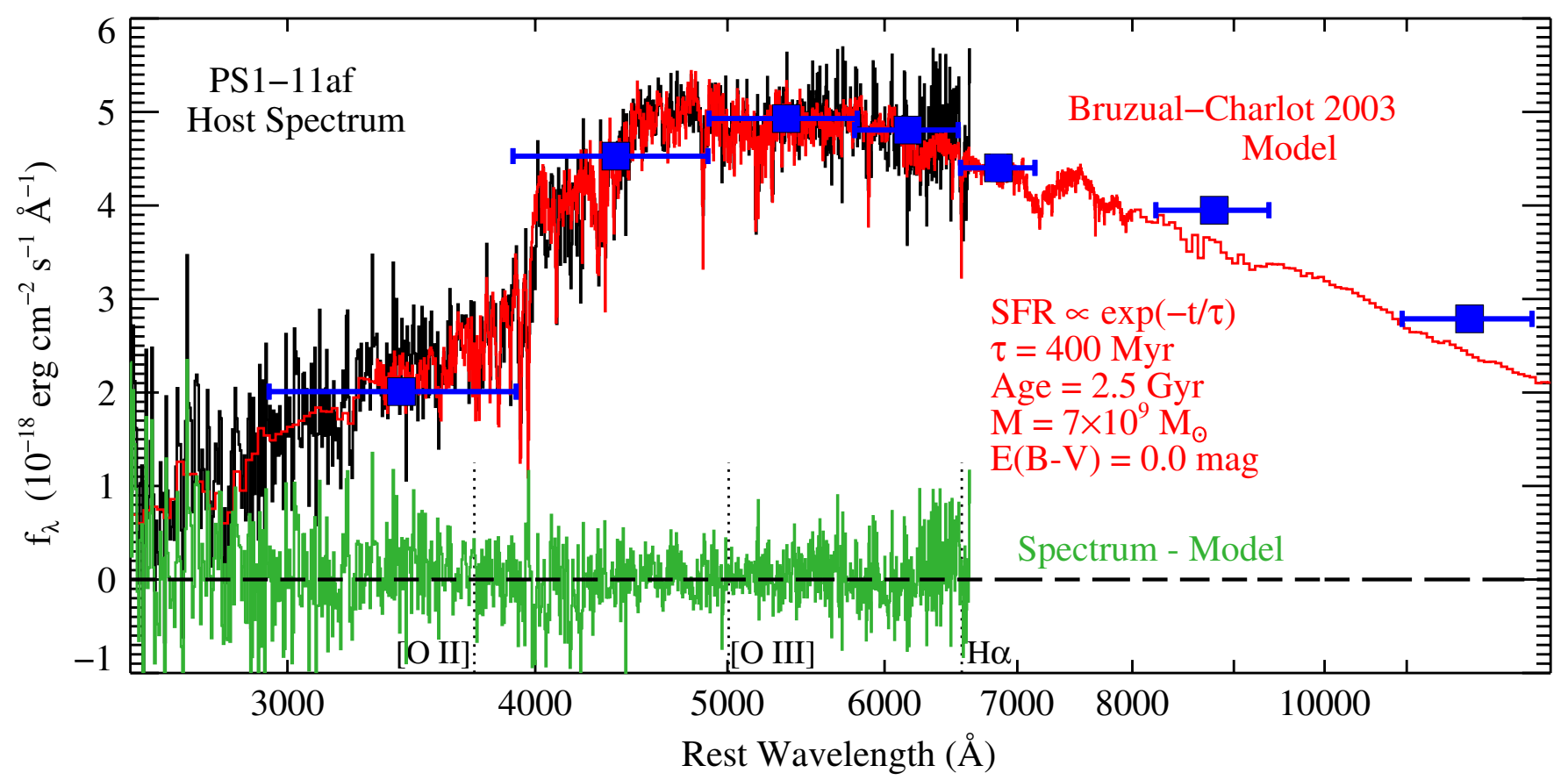

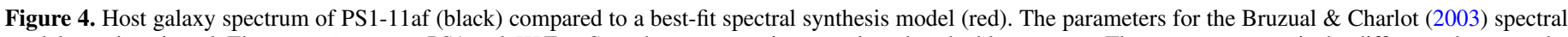

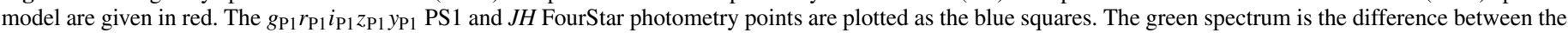

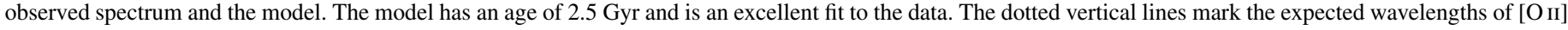
$\lambda 3727,[\mathrm{O} I I I] \lambda 5007$, and $\mathrm{H} \alpha$, which would be signatures of either star formation or AGN activity. No emission from any line is detected.

(A color version of this figure is available in the online journal.)

Table 4

PS1-11af Host Galaxy Photometry

\begin{tabular}{|c|c|c|c|c|c|c|c|}
\hline \multirow[t]{2}{*}{ Filter } & \multirow{2}{*}{$\begin{array}{c}\text { Observed } \\
\text { Wavelength }(\AA)\end{array}$} & \multirow{2}{*}{$\begin{array}{c}\text { Rest-frame } \\
\text { Wavelength }(\AA)\end{array}$} & \multicolumn{2}{|c|}{ 1". 15 Aperture } & \multicolumn{2}{|c|}{$3^{\prime \prime}$ Aperture } & \multirow[t]{2}{*}{ Instrument } \\
\hline & & & AB Mag ${ }^{\mathrm{a}}$ & Error & AB Mag ${ }^{\mathrm{a}}$ & Error & \\
\hline FUV & 1539 & 1095 & $\ldots$ & $\ldots$ & $>24.4^{b}$ & $\ldots$ & $G A L E X$ \\
\hline NUV & 2316 & 1649 & $\ldots$ & $\ldots$ & $>24.3$ & $\ldots$ & $G A L E X$ \\
\hline$g_{\mathrm{P} 1}$ & 4825 & 3424 & 23.42 & 0.04 & 22.68 & 0.04 & PS1 \\
\hline$r_{\mathrm{P} 1}$ & 6170 & 4393 & 22.00 & 0.02 & 21.35 & 0.02 & PS1 \\
\hline$i_{\mathrm{P} 1}$ & 7520 & 5354 & 21.48 & 0.02 & 20.87 & 0.02 & PS1 \\
\hline$z_{\mathrm{P} 1}$ & 8660 & 6165 & 21.20 & 0.02 & 20.60 & 0.02 & PS1 \\
\hline$y_{\mathrm{P} 1}$ & 9620 & 6849 & 21.07 & 0.03 & 20.45 & 0.04 & PS1 \\
\hline$J$ & 12360 & 8798 & 20.64 & 0.03 & 20.02 & 0.03 & FourStar \\
\hline$H$ & 16620 & 11830 & 20.38 & 0.02 & 19.86 & 0.02 & FourStar \\
\hline
\end{tabular}

Notes.

a Corrected for Galactic extinction.

b Upper limits are $5 \sigma$.

The FourStar data were taken under significantly better seeing $(\mathrm{FWHM} \approx 0$.'75) than the PS1 images. The core of the host is not well resolved, so the quoted aperture photometry was calculated after convolving the FourStar data with a Gaussian to approximately match the seeing in the PS1 templates and make the narrow apertures more directly comparable between instruments. We stack the pre-outburst GALEX photometry and set upper limits on the host galaxy flux in the UV.

In addition to the photometry, we have obtained about $4 \mathrm{hr}$ of spectroscopy of the host galaxy, as described in Section 2.2. The resolutions of the $\mathrm{BC}$ and LDSS3 spectra are similar, so we combined all of the spectra by rebinning to a common wavelength scale and performing a weighted average over the overlap regions. We plot this combined spectrum in Figure 4, after scaling to the host photometry in the narrow apertures because they more closely match the size of the longslit spectroscopic apertures.

\subsection{Limits on Current Star Formation}

It is obvious from the strength of the $4000 \AA$ break that the spectrum shown in Figure 4 is that of an older stellar population. The Balmer lines are only present in absorption, and there are no emission lines of any type, while absorption lines from several metals are strong. We cross-correlate this spectrum with the single stellar population age models of Bruzual \& Charlot (2003). All models that are good fits have ages $>1$ Gyr, with the best fit at solar metallicity being $2.5 \mathrm{Gyr}$. The best-fit cross correlations give a precise redshift of $z=0.4046 \pm 0.0001$.

We use the FAST program of Kriek et al. (2009) to simultaneously fit the spectrum and photometry to a suite of solar metallicity stellar population models from Bruzual \& Charlot (2003) with more complicated star formation histories. The best fit is overplotted in red and uses an exponentially declining star formation rate (SFR) with an e-folding timescale of $400 \mathrm{Myr}$ 
and an age of 2.5 Gyr. Only the $r_{\mathrm{P} 1}$ and $i_{\mathrm{P} 1}$ points were used to scale the spectrum, so the good agreement in the other bands is reassuring. The total stellar mass is $7 \times 10^{9} M_{\odot}$ when scaled just to the photometry in the inner aperture. The photometry in the outer aperture is $\sim 0.6$ mag brighter, so if all of the flux is assigned to the host, then the total stellar mass is closer to $1.2 \times$ $10^{10} M_{\odot}$.

The difference spectrum shown at the bottom of Figure 4 shows no strong deviations between the model and the observed spectrum. In particular, no emission lines are visible. We use the difference spectrum and observed errors to set $3 \sigma$ upper limits on the ([O II $] \lambda 3727, \mathrm{H} \alpha)$ emission fluxes of $(5.2,4.8) \times$ $10^{-18} \mathrm{erg} \mathrm{cm}^{-2} \mathrm{~s}^{-1}$ in $10 \AA$ bins around the rest wavelengths of the lines. According to the calibration of Kennicutt (1998), these correspond to upper limits on the SFR of the host galaxy of $(0.04,0.02) M_{\odot} \mathrm{yr}^{-1}$. Similarly, we also use the non-detections in the UV by GALEX and the calibrations of Kennicutt (1998) to set $5 \sigma$ upper limits on the SFR of $0.8 M_{\odot} \mathrm{yr}^{-1}$. Combined, these indicate that there is no sign of star formation or any young stellar population in the host galaxy at the position of PS1-11af. In addition, we inspect our spectra of the host galaxy obtained at the PA of $-10^{\circ}$. While the surface brightness of the extension is too low to obtain spectra with a good $\mathrm{S} / \mathrm{N}$ in our limited exposure time, inspection of the two-dimensional frames shows no obvious emission lines.

\subsection{Evidence Against an AGN Host}

An outburst in a persistently accreting AGN could masquerade as a true transient. However, the non-detection in the UV by GALEX argues strongly against the presence of a luminous unobscured AGN in the nucleus. The stacked nondetections in the GALEX bands correspond to absolute magnitudes $M_{\mathrm{UV}}>-17 \mathrm{mag}$ or UV continuum luminosities $v L_{v} \lesssim 6 \times 10^{42} \mathrm{erg} \mathrm{s}^{-1}$. Also, the difference spectrum at the bottom of Figure 4 exhibits no emission lines from the broadline region (BLR) of an AGN. The spectra of both obscured and unobscured AGN could also manifest strong forbidden emission lines from a narrow-line region (NLR), but those are not present either. The $3 \sigma$ limit on the [O III] $\lambda 5007$ luminosity is $<1.4 \times 10^{39} \mathrm{erg} \mathrm{s}^{-1}$, which falls below any of the optically selected type II AGNs at similar redshifts in the zCOSMOS survey (Bongiorno et al. 2010). However, we cannot exclude the possibility of a low-luminosity AGN, as samples in the local universe extend to significantly lower emission-line luminosities than our limits for the host of PS1-11af (Ho 2008; Hao et al. 2005). All of these upper limits would be made weaker by the presence of strong dust obscuration in the nucleus, but the blue color of the transient (Figure 3; see below) is incompatible with a large dust column along the line of sight.

The deep non-detection of the host galaxy in the UV prior to the detection of PS1-11af also represents a strong argument against normal accretion rate fluctuations in an AGN. As described by Gezari et al. (2012), quasars and AGN exhibit variability of a lower amplitude in GALEX observations than the $>2.5$ mag increase in brightness of PS1-11af relative to quiescence. In the full GALEX TDS observations (Gezari et al. 2013), most sources with such large amplitude NUV flares are classified as cataclysmic variables or M-dwarf flares. The only extragalactic sources having similarly large amplitude flares after non-detections in quiescence were the TDE PS1-10jh and a few SNe (Gezari et al. 2012).

Another possibility is that PS1-11af is an outburst from a blazar (Urry \& Padovani 1995), but we would expect to

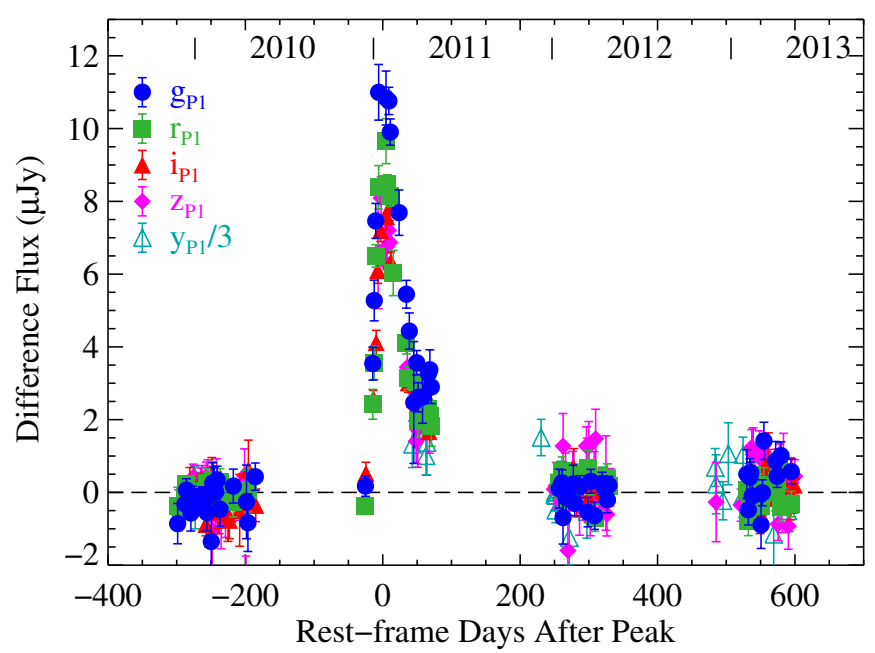

Figure 5. Long-term difference flux light curve of PS1-11af in five filters. The calendar years of observations are labeled across the top. The $y_{\mathrm{P} 1}$ light curve has been divided by three for clarity because the observations are shallower than in the other bands so the typical scatter is larger. This light curve was constructed using a template image composed of an average of several of these same epochs of observation, which means that the zeropoint of the flux scale has an arbitrary constant offset level that we set to zero in the first year.

(A color version of this figure is available in the online journal.)

detect a blazar in the radio. Near the peak of the outburst, PS1-11af had a $V$-band magnitude of $\sim 21.5$, corresponding to $v L_{v} \approx 3 \times 10^{43} \mathrm{erg} \mathrm{s}^{-1}$. The unified blazar SEDs of Fossati et al. (1998) predict radio fluxes in the range $0.4-15 \mathrm{mJy}$ for different ranges of radio loudness. Even the faintest of these is $\sim 8$ times brighter than our $3 \sigma$ upper limit in the first epoch. The broad UV absorption features of PS1-11af would also be unexpected in the synchrotron-dominated spectra of a blazar.

As a final check against AGN-like variability, we present difference light curves in five filters for PS1-11af from all four years of PS1 observations obtained to date in Figure 5. This light curve shows no sign of variability or any long-term trend during the other three observing seasons to date. The host galaxy was also observed by SDSS a decade prior to our detection of PS111af (object ID: SDSS J095726.82 + 031400.9), and the model magnitudes reported by Ahn et al. (2012) from 2001 February 20 are consistent within $1 \sigma$ of our PS 1 photometry in the large aperture (Table 4).

The fast rise in $g_{\mathrm{P} 1}$ of PS1-11af is especially notable in this context. The host flux in the narrow aperture was $g_{\mathrm{P} 1}=$ $23.42 \mathrm{mag}$, but PS1-11af rose on a timescale of less than a month to a peak of $g_{\mathrm{P} 1} \approx 21.5 \mathrm{mag}$, implying a rise of more than 2 mag (the quiescent flux is clearly dominated by star light; Figure 4). The characteristic amplitude of quasar photometric variability on these timescales is only $\sim 0.1 \mathrm{mag}$, with the amplitude of variability increasing on longer timescales (e.g., Webb \& Malkan 2000; MacLeod et al. 2012a). This is very different from the long-term PS1-11af light curve, which is consistent with a constant in the observing seasons lacking the transient.

\section{EVOLUTION OF PS1-11af}

The light curve of PS1-11af exhibits a relatively rapid rise of $\sim 2$ mag from the first detections in $g_{\mathrm{P} 1} r_{\mathrm{P} 1}$ to the peak 14.5 rest-frame days later. The subsequent decline from maximum light was much slower, with a decline of $\lesssim 2$ mag by the time of our final GMOS observations at nearly Day +100 (this notation refers to the phase of the object in rest-frame days relative to 


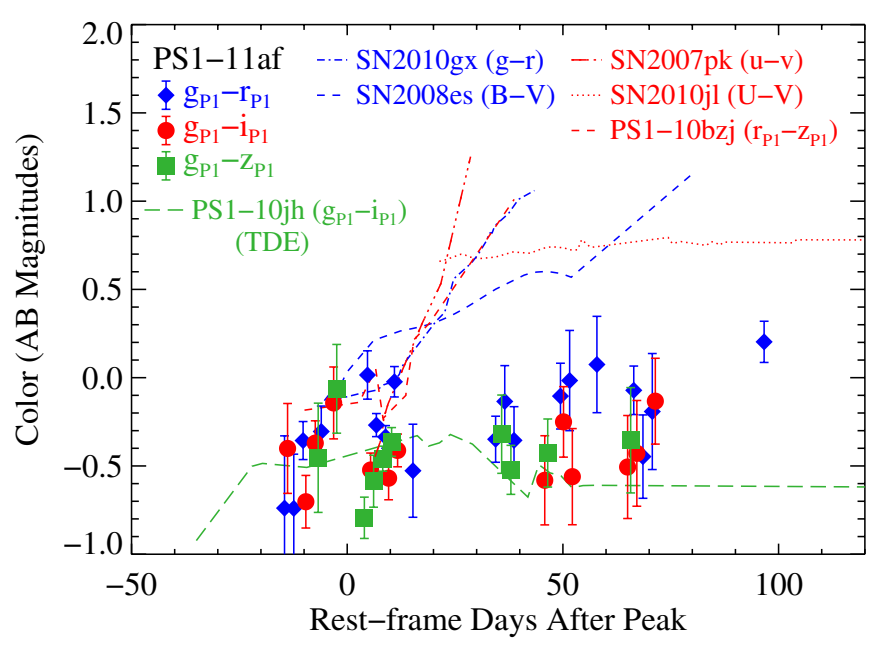

Figure 6. Observed color evolution of PS1-11af compared to several SNe and one TDE. The comparison objects are labeled with the observed filters used for the color curves. Note the blue and relatively constant colors of PS1-11af compared to the SNe, which rapidly evolve redward. The objects and the restframe wavelengths of the plotted filters are: SN 2010gx/PTF $10 \mathrm{cwr}(\mathrm{g}, 3770 \AA$; $r, 4975 \AA$; Pastorello et al. 2010; Quimby et al. 2011), SN 2008es (B, $3600 \AA$; $V, 4490 \AA$; Miller et al. 2009; Gezari et al. 2009a), SN 2007pk (u, $3465 \AA$; $v$, $5468 \AA$; Pritchard et al. 2012), SN 2010jl (U, $3430 \AA$; $V, 5410 \AA$; Zhang et al. 2012), PS1-10bzj ( $r_{\mathrm{P} 1}, 3740 \AA$; $z_{\mathrm{P} 1}, 5250 \AA$; Lunnan et al. 2013), and PS1-10jh $\left(g_{\mathrm{P} 1}, 4110 \AA ; i_{\mathrm{P} 1}, 6430 \AA\right.$; Gezari et al. 2012).

(A color version of this figure is available in the online journal.)

maximum light). This basic light curve shape was present in all filters (Figure 2), which implies very little color evolution.

The weak evolution of the rather blue SED is one of the most important clues to the nature of PS1-11af, so we examine it in detail. We present the observed colors in the PS1 filters in Figure 6. We calculate $g_{\mathrm{P} 1}-r_{\mathrm{P} 1}$ data points only when we have $g_{\mathrm{P} 1}$ and $r_{\mathrm{P} 1}$ observations on the same night. The other filters were generally not obtained simultaneously, so we interpolate the less-noisy $g_{\mathrm{P} 1}$ light curve to the dates of observation of the $i_{\mathrm{P} 1}$ and $z_{\mathrm{P} 1}$ data using low-order polynomials. We determine the uncertainties in the derived colors by repeatedly re-fitting the observed data points after adjusting by Monte Carlo resamplings of the errors. We also apply this procedure below whenever we need to interpolate the photometry to common epochs.

The $g_{\mathrm{P} 1}-i_{\mathrm{P} 1}$ and $g_{\mathrm{P} 1}-z_{\mathrm{P} 1}$ data points in Figure 6 are consistent with constant values from Day -10 to +70 . The $g_{\mathrm{P} 1}-r_{\mathrm{P} 1}$ color is similarly constant over this time interval; however, the final point near Day +97 is redder, and there is a hint of a bluer color at earlier times. We compare the PS1-11af data to several SNe as well as the TDE PS1-10jh (Gezari et al. 2012). The color curves for the comparison objects are color coded so that lines of a given color correspond to approximately similar rest-frame wavelengths as the PS1-11af data points of the same color. This largely eliminates the need to apply uncertain $K$-corrections. For example, $r_{\mathrm{P} 1}$ and $z_{\mathrm{P} 1}$ at the redshift of PS1-10bzj ( $z=0.650$; Lunnan et al. 2013) are at rest-frame wavelengths of 3740 and $5250 \AA$, respectively, which are close to $g_{\mathrm{P} 1}$ and $i_{\mathrm{P} 1}$ at the redshift of PS1-11af (cf. Table 4), so both are plotted in red. The key point is that regardless of any extinction or $K$-correction, the $\mathrm{SNe}$ are only briefly as blue as PS1-11 af and rapidly become redder with time, while the TDE PS1-10jh (Gezari et al. 2012) has a similarly blue color that does not strongly evolve with time. We discuss the implications of this observation in more detail in Section 5.

We now investigate the SED of PS1-11af by interpolating the observed photometry to four common epochs, which we display

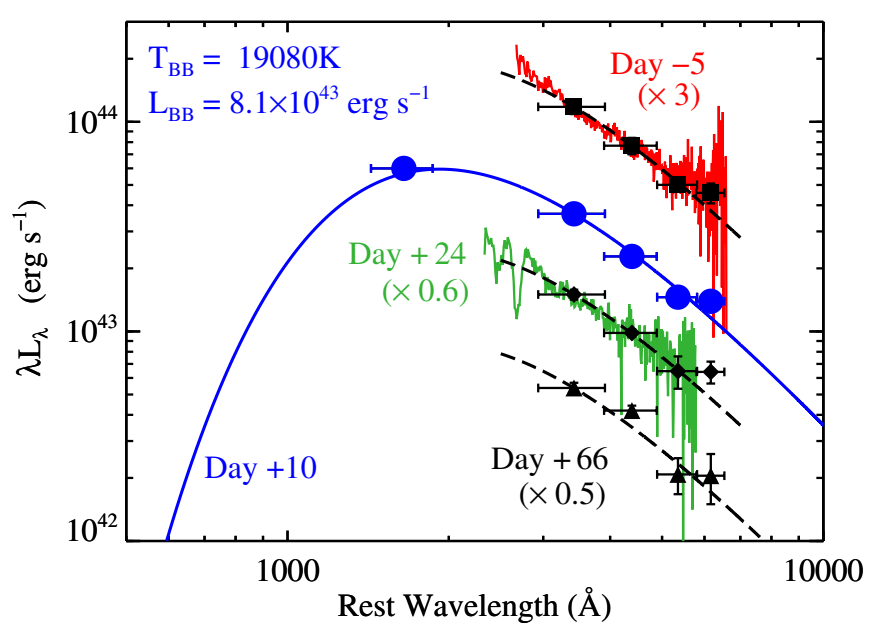

Figure 7. SED evolution of PS1-11af. The photometry has been interpolated to four common epochs. The data points with blue circles come from Day +10 , near the beginning of the GALEX observations. The other epochs have been offset for clarity by the multiplicative factors listed in parentheses. The dashed lines at the other three epochs represent the same BB fit as the thick blue line but are scaled in flux to match the $g_{\mathrm{P} 1}$ point at the appropriate epoch. This BB fit continues to be a good fit for the data at other epochs, indicating a lack of strong SED evolution. The red and green lines are the host galaxy subtracted spectra from our first two epochs of spectroscopy.

(A color version of this figure is available in the online journal.)

in Figure 7. The GALEX data provide an important constraint on the SED but are limited in phase coverage. Therefore, we first examine the SED on Day +10 , near the beginning of the GALEX observations. We fit a single-temperature $\mathrm{BB}$ to the NUV through $z_{\mathrm{P} 1}$ SED and overplot the fit as the blue solid line (Figure 7). The best fit has $T_{\mathrm{BB}}=19,080 \pm 750 \mathrm{~K}$, with a luminosity $\left(L_{\mathrm{BB}}\right)$ of $8.1 \times 10^{43} \mathrm{erg} \mathrm{s}^{-1}$. In addition, we plot the $g_{\mathrm{P} 1} r_{\mathrm{P} 1} i_{\mathrm{P} 1} z_{\mathrm{P} 1}$ photometry at the epochs of our first two spectra (Days -5 and +24 ) and the date of our final $z_{\mathrm{P} 1}$ detection (Day +66). The solid dashed lines overplotted on the photometry for each epoch are the same BB fit scaled to the $g_{\mathrm{P} 1}$ flux on each date. The same BB fit from the GALEX epoch is also an adequate fit to the $g_{\mathrm{P} 1} r_{\mathrm{P} 1} i_{\mathrm{P} 1} z_{\mathrm{P} 1}$ SEDs separated by 71 rest-frame days.

Although the PS1 data are largely on the Rayleigh-Jeans tail of the SED and we lack NUV observations for most of the light curve, we still obtain useful constraints on the SED using our optical data. We interpolate the $i_{\mathrm{P} 1}$ and $z_{\mathrm{P} 1}$ light curves to the dates on which we have both $g_{\mathrm{P} 1}$ and $r_{\mathrm{P} 1}$ data. Our BB fits to the available photometry are shown in the middle and bottom panels of Figure 8. As we inferred from the color evolution, the best-fit $T_{\mathrm{BB}}$ is consistent with a constant from Days -10 to +70 . The NUV+PS1 fit from Figure 7 is shown as a blue circle and is consistent with our optical-only fits.

We have no a priori reason to believe that the SED of PS111 af should be well approximated by a single-temperature BB, so we also fit the $g_{\mathrm{P} 1} r_{\mathrm{P} 1} i_{\mathrm{P} 1} z_{\mathrm{P} 1}$ photometry with power laws of the form $f_{v} \propto v^{\alpha}$ and plot the best-fit power-law indices in the top panel of Figure 8. These fits again demonstrate a lack of significant SED evolution during the course of our observations. A weighted average of the fits to all four filters (diamonds in the top panel of Figure 8) gives $\alpha=0.73 \pm 0.05$.

This is notable for being significantly bluer than AGN SEDs over this wavelength range. Composite quasar templates have mean values for $\alpha$ in the range -0.32 to -0.50 for the NUV-optical continuum depending on the selection criteria (Brotherton et al. 2001; Vanden Berk et al. 2001). These values represent average quasar SEDs, but Wilhite et al. (2005) isolated 


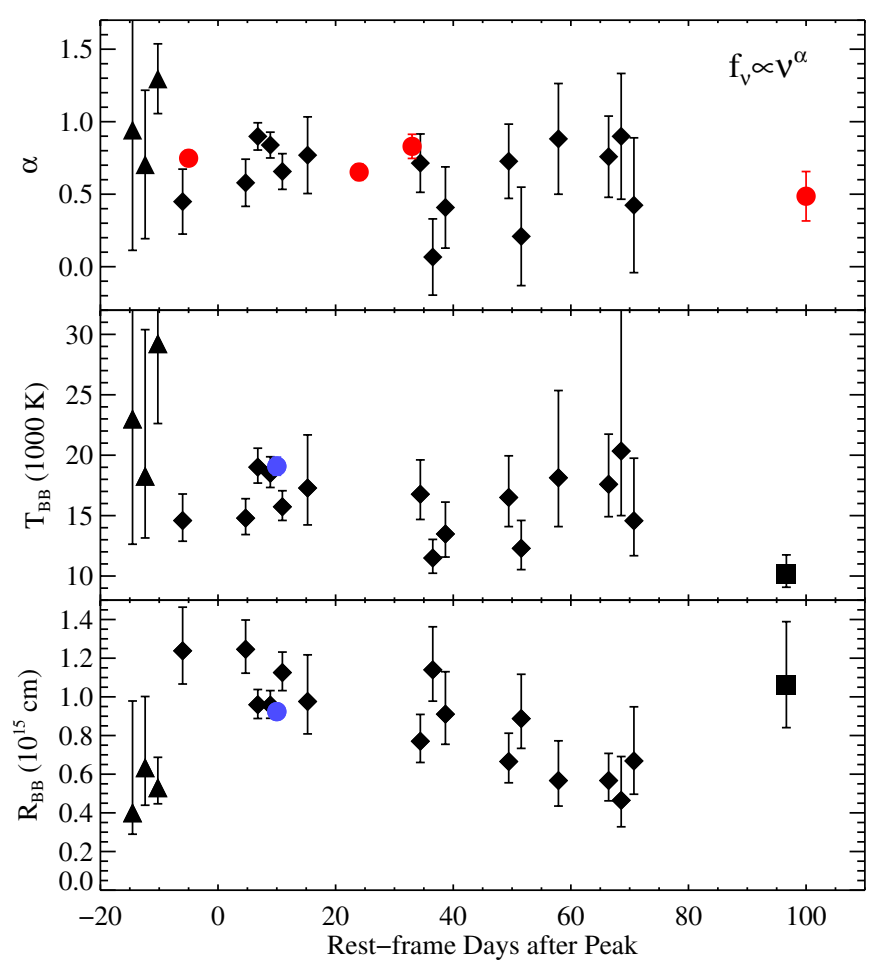

Figure 8. Power law (top) and BB (middle, bottom) fits to the optical photometry of PS1-11af. The black diamonds mark fits to $g_{\mathrm{P} 1} r_{\mathrm{P} 1} i_{\mathrm{P} 1} z_{\mathrm{P} 1}$, while triangles are fits to $g_{\mathrm{P} 1} r_{\mathrm{P} 1} i_{\mathrm{P} 1}$ (before the start of the $z_{\mathrm{P} 1}$ observations), and the square is a fit to the late-time $g^{\prime} r^{\prime}$ data only. The blue circles in the middle and bottom panels are from the BB fit to GALEX + PS1 data shown in Figure 7. The red circles in the top panel are fits to the galaxy-subtracted spectra from Section 4.1.

(A color version of this figure is available in the online journal.)

the variable component of the spectra from multiepoch SDSS spectroscopy. This variable component is bluer than the average but still has an NUV-optical slope of only $\alpha \approx 0$. This is also not as blue as PS1-11af and provides additional evidence that it is not a result of normal AGN variability. We note that PS1-11af is also bluer than the canonical $\alpha=1 / 3$ value for multicolor $\mathrm{BB}$ disk emission (Pringle \& Rees 1972). In a pure AGN accretion disk model, this implies that the SED of PS1-11af does not include as much emission from the cooler large radii as in normal AGN disks. A simple explanation is that TDE accretion disks are smaller than those of AGN, although below we discuss the reasons to believe that the optical emission of PS1-11af does not directly originate in a disk.

\subsection{Galaxy-subtracted Spectra}

The observed spectra of PS1-11af shown in Figure 3 exhibit $\mathrm{Ca}$ II $\mathrm{H}+\mathrm{K}$ absorption from the host galaxy as well as several undulations that correspond to similar features in the host spectrum (Figure 4). A comparison of the host galaxy photometry (Table 4) and the transient light curve (Table 1) reveals that even near maximum light we should expect a significant contribution from the host galaxy to the observed fluxes, particularly at the redder wavelengths. We now use the information about the host galaxy from Section 3.1 and the photometric properties of PS111 af from Section 4 to subtract the host galaxy contribution from the observed spectra and isolate the spectrum of the transient.

The host galaxy is spatially resolved while the transient is not, so seeing variations mean that the amount of galaxy light relative to the transient in the spectroscopic slit aperture cannot be reliably determined directly from the photometry. Instead, we fit the observed spectra to model the host contribution. We

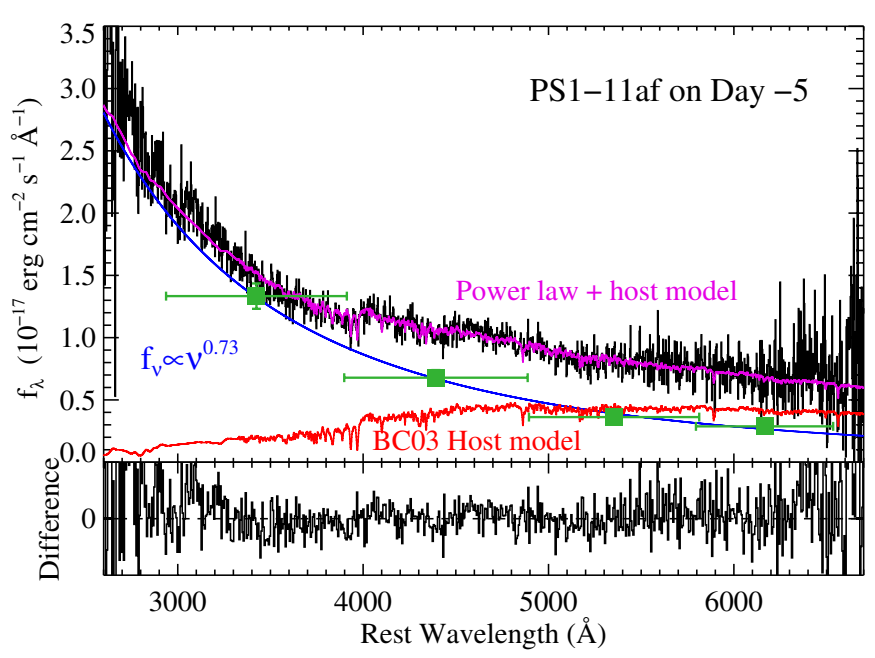

Figure 9. Demonstration of our procedure for determining the proper amount of galaxy light to subtract from each epoch of spectroscopy. The observed spectrum (black) is modeled as the sum (magenta) of our best-fit host galaxy model (red; Figure 4) and a power-law continuum with a fixed slope (blue). The difference between the observed spectrum and the sum is shown in the bottom panel. The green squares are the $g_{\mathrm{P} 1} r_{\mathrm{P} 1} i_{\mathrm{P} 1} z_{\mathrm{P} 1}$ photometry of the transient interpolated to the date of the spectrum. The photometry was not used to scale the power-law continuum in the fits, so the excellent agreement with the derived scale factor indicates that our spectrophotometry is reliable.

(A color version of this figure is available in the online journal.)

initially model each epoch of spectroscopy as a linear sum of the best-fit host model from Section 3.1 and a $\mathrm{BB}$ with $T_{\mathrm{BB}}$ equal to the value determined above from the GALEX+PS1 SED fits and determine the best-fit scale factors for each component. We previously used a similar procedure to subtract the host galaxy of PS1-10jh from its spectra (Gezari et al. 2012). We then repeat our subtraction procedure using a power-law continuum with $\alpha=0.73$ and find statistically superior fits. At each epoch, the scaling factors for the amplitudes of the host galaxy model are identical for the $\mathrm{BB}$ and power law models to within $1 \%-3 \%$, indicating that our subtraction procedure is not sensitive to this choice for the transient SED, although the scaling factors do change if $T_{\mathrm{BB}}$ or $\alpha$ are varied. An example fit to the first PS1-11af spectrum is shown in Figure 9. The depths of the absorption lines and amplitudes of the continuum undulations due to the host in the model spectrum (magenta line) match those in the data very well.

Several features of the fitting procedure are worth noting. The first is that we do not include any constraint from the observed photometry (except implicitly through our choice of the power-law index or $T_{\mathrm{BB}}$ ) due to the possibility that our absolute spectrophotometry is unreliable because of clouds or slit losses. However, the green squares overplotted on Figure 9 represent the $g_{\mathrm{P} 1} r_{\mathrm{P} 1} i_{\mathrm{P} 1} z_{\mathrm{P} 1}$ photometry interpolated to the date of the spectrum. The excellent agreement of the photometry with both the amplitude and color of the scaled power law is apparent. This gives us confidence that the spectrophotometry is correct and that our procedure to scale and subtract the host is working satisfactorily. This can be seen in another way in Figure 7, where we overplot the galaxy-subtracted spectra on Days -5 and +24 along with the photometry. Although we apply the indicated multiplicative offsets for clarity, no relative normalization factors were applied between the photometry and the spectroscopy at the same epoch. Again, the colors and normalization of the galaxy-subtracted spectra are in excellent agreement with the photometry. 
Second, the spectra on the first two epochs exhibit a blue excess relative to the host plus $\mathrm{BB}$ model that can be seen at wavelengths below $\sim 3200 \AA$ in Figure 7 . This is not simply a consequence of using an incorrect (too low) $T_{\mathrm{BB}}$. If we allow $T_{\mathrm{BB}}$ to vary in our fits, we can find better fits to match the overall shape of the observed spectra if $T_{\mathrm{BB}}$ is in the range 25,000-30,000 K. However, the fits compensate for the bluer assumed BB color by increasing the amplitude of the host contribution to match the red flux. After subtraction of the new best-fit host contribution, the derived transient spectrum then has a color that is too blue relative to the colors measured from the photometry. Equivalently, the values for $T_{\mathrm{BB}}$ allowed by the optical photometry (Figure 8) are lower than those required to remove the blue/UV excess flux. The simplest explanation is that the blue excess is real and the SED of PS1-11af is not that of a pure single-temperature $\mathrm{BB}$. Therefore, in the remainder of this paper, we use the statistically preferred power-law model to determine the proper scaling factors for the host galaxy. We do not claim that the true SED is a power law, just that a power law is a better approximation over the limited wavelength range of our spectra for the purpose of scaling and removing the host contribution. We also note that the shape of the excess is inconsistent with Balmer continuum emission, which would be expected to peak near $3650 \AA$.

Another consideration is that we subtract the spectralsynthesis model for the host galaxy spectrum but the real spectrum could be different. The excellent fit of the host model in Figure 4 demonstrates that any such error is small. We repeat the subtraction procedure using our actual host galaxy spectrum and find no significant difference in the subtracted spectra, including the presence of the UV excess in the fits with a BB continuum. However, the subtracted spectra are noticeably noisier, especially at shorter wavelengths, so we use the model-subtracted spectra in all subsequent analysis.

After determining the relative amplitude of the host galaxy contribution for each epoch, ${ }^{19}$ we subtract the appropriately scaled host galaxy model and present the resulting spectral sequence in Figure 10. The spectra of PS1-11af are very blue, with the Day -5 spectrum being well fit by a power law with $\alpha=0.75 \pm 0.02$, consistent with the values measured from the photometry, as shown in the top panel of Figure 8. No clear emission features (either narrow or broad) are present in any of our spectra, unlike the broad He II emission seen from PS1-10jh (Gezari et al. 2012) or the broad $\mathrm{H} \alpha$ emission detected from SDSS TDE2 (van Velzen et al. 2011a). The Day + 24 spectrum exhibits two strong absorption features shortward of $3000 \AA$, with minima near 2450 and $2680 \AA$. The reddest of these is clearly not present on Day -5 in our only other spectrum with overlapping wavelength coverage. We note that these features are definitely real (they are apparent even in the two-dimensional spectral frames) and are largely unaffected by any details of the host galaxy subtraction procedure. The host galaxy has very little contribution at these wavelengths (e.g., Figure 9), and the features are present prior to host subtraction in Figure 3.

\section{IS PS1-11af A SN?}

PS1-11af exhibits strong, broad UV absorption features in the Day +24 BC spectrum (Figure 10) that are strikingly similar to the P-Cygni absorptions present in $\mathrm{SN}$ atmospheres. In this section, we demonstrate that PS1-11af is unlike any known SN

\footnotetext{
19 We exclude the regions around the strong absorption minima on Day +24
} from the fit.

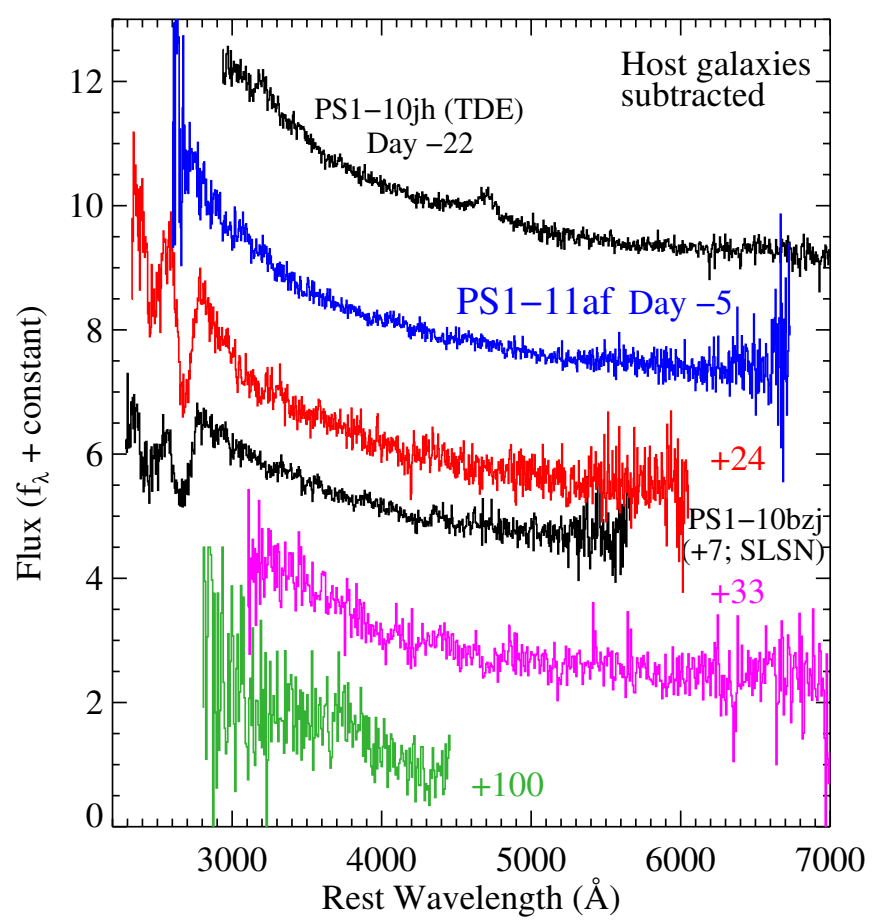

Figure 10. Spectra of PS1-11 af after subtraction of the host-galaxy contribution. Note the very blue color of the spectra, with the Day +24 spectrum having deep UV absorption features. The black spectrum in the middle is the $+7 \mathrm{~d}$ spectrum of PS1-10bzj, which also shows similar features (Lunnan et al. 2013). The top black spectrum is the TDE PS1-10jh at Day -22 (Gezari et al. 2012). Note the broad emission lines from He II $\lambda 4686$ and $\lambda 3203$ in that object, which are not present in any of the PS1-11af spectra.

(A color version of this figure is available in the online journal.)

and appears to be inconsistent with the expectations for any plausible $\mathrm{SN}$ or explosive transient.

We start with the observation that our observed wavelength coverage for PS1-11af includes the Balmer lines expected in $\mathrm{SNe}$ II as well as the diagnostic He I lines of $\mathrm{SNe} \mathrm{Ib}$ ( $\lambda 5876$ would be strongest), and none of these are present. The spectra also lack the optical features due to Fe II, Ca II, and the intermediate-mass elements found in both SNe Ia and normal SNe Ic (Filippenko 1997). Instead, the combination of an optical continuum having at most weak features with strong $\mathrm{UV}$ absorptions is at least qualitatively similar to the spectra of hydrogen-poor superluminous SNe (SLSNe).

The transient SCP06F6 exhibited a triplet of absorption features between 2000 and $3000 \AA$ (Barbary et al. 2009). The two longer-wavelength ones were first identified by Quimby et al. (2011) as Si III and Mg II, although spectral modeling indicates that some Fe II may contribute (Lunnan et al. 2013). We examine the published spectra of SLSNe, and the best match we find is to the Day +7 spectrum of PS1-10bzj, which we plot as a comparison in Figure 10 (Lunnan et al. 2013). Most other SLSNe with optical spectroscopy at sufficiently early epochs to match the blue colors of PS1-11af exhibit a series of absorption features in the blue part of the optical that have been identified as being due to O II, the strongest being a "W"-shaped absorption near $4300 \AA$ (e.g., Quimby et al. 2007, 2011; Pastorello et al. 2010; Chomiuk et al. 2011). Other objects show Fe II features in the rest-frame optical (e.g., Inserra et al. 2013) and even evolve to resemble normal SNe Ic (Pastorello et al. 2010; Quimby et al. 2011). The spectra of PS1-11af are instead devoid of strong features at optical wavelengths at all available epochs. 


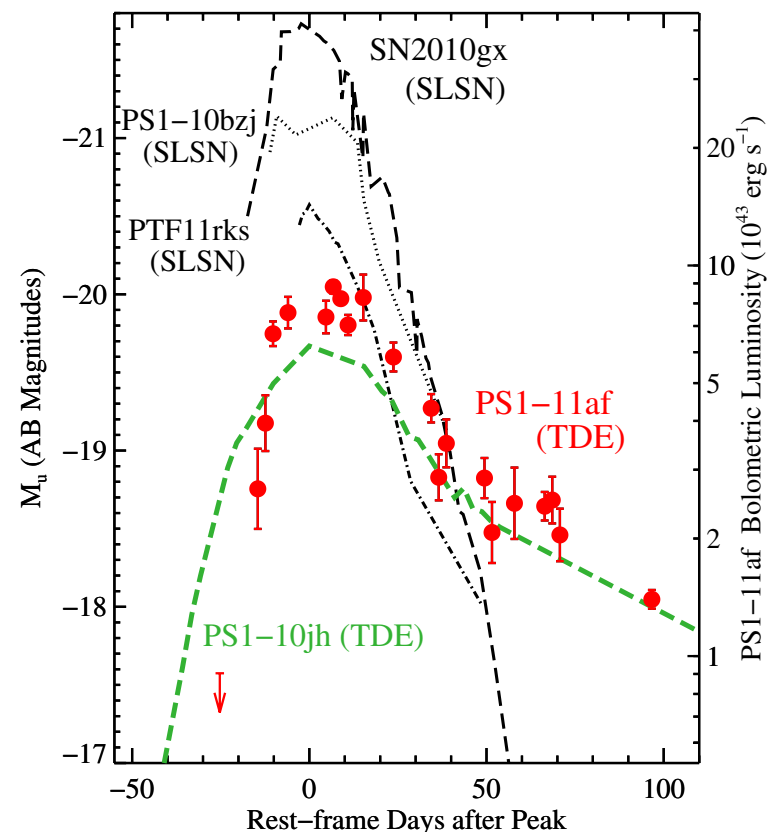

Figure 11. Light curve of PS1-11af compared to SLSNe and PS1-10jh in filters with rest-frame wavelengths near $u$ band. The PS1-11af data points are the $g_{\mathrm{P} 1}$ light curve $\left(\lambda_{\text {rest }}=3425 \AA\right)$ and the bolometric luminosity scale on the right axis represents this light curve multiplied by a bolometric correction factor determined from the Day + 10 SED (Figure 7). We caution that due to differing SEDs and bolometric correction factors, this luminosity scale only applies to PS1-11af. The other objects, along with the plotted filters and restframe wavelengths, are: PS1-10jh (green dashed line; $g_{\mathrm{P} 1}, 4110 \AA$; Gezari et al. 2012), SN 2010gx (black dashed line; $g / B$, 3546/3770 A; Pastorello et al. 2010; Quimby et al. 2011), PS1-10bzj (black dotted line; $r_{\mathrm{P} 1}, 3740 \AA$ A Lunnan et al. 2013), and PTF11rks (black dot-dashed line; $g, 3900 \AA$; Inserra et al. 2013). Arrows denote $3 \sigma$ upper limits.

(A color version of this figure is available in the online journal.)

We also compare the light curve of PS1-11af to a few of these SLSNe in Figure 11. In each case, we select filters with restframe wavelengths near $u$ band and correct for the distance modulus and cosmic expansion without performing a full $K$-correction due to the substantial uncertainties involved (e.g., PTF11rks, which has a rather red, but poorly sampled, observedframe $u-g$ color; Inserra et al. 2013). The bulk of the hydrogenpoor SLSNe published to date are significantly more luminous in $u$ than PS1-11af (e.g., Quimby et al. 2007, 2011; Chomiuk et al. 2011). We choose three SLSNe at the lower end of the luminosity distribution for comparison, but which are still more luminous in $u$ than PS1-11af. Note that the larger bolometric correction for the bluer SED of PS1-11 af makes its peak bolometric luminosity $\left(\sim 8 \times 10^{43} \mathrm{erg} \mathrm{s}^{-1}\right)$ closer to that of the SLSNe $\left(\gtrsim 10^{44} \mathrm{erg} \mathrm{s}^{-1}\right)$ than a simple comparison of $M_{u}$ implies.

In addition, PS1-11af took $\sim 100 \mathrm{~d}$ to decline two magnitudes from peak while the comparison SLSNe declined that much from peak in only 30-40 d. By our $r_{\mathrm{P} 1}$ photometric point on Day +97, PS1-11af was still near $M_{g} \approx-18.25 \mathrm{mag}$, significantly more luminous than the objects in the sample of Inserra et al. (2013), with only PTF09cnd among the published SLSNe being similarly luminous at that late epoch, and it peaked at $M_{u} \approx-22 \mathrm{mag}$ (Quimby et al. 2011). The faster light curve evolution of the SLSNe is a combination of actual faster bolometric declines along with cooling ejecta leading to a smaller fraction of the flux being emitted at such blue wavelengths.

This implies that the spectral correspondence may be coincidental. The SLSNe generally show some spectral evolution over time, so the similarity may not necessarily hold true at other times. Even PS1-10bzj had a spectrum on Day +15 (only $\sim 8 \mathrm{~d}$ after the plotted one) that had evolved in the UV and developed stronger optical lines (Lunnan et al. 2013), no longer appearing as similar to PS1-11af. The optical spectra of the SLSN 2010gx started to resemble normal SNe Ic by Day +21 (Pastorello et al. 2010), an epoch prior to that of our BC spectrum of PS1-11af with the UV features. Other, more luminous, SLSNe do evolve more slowly spectroscopically (e.g., SCP06F6; Barbary et al. 2009). Unlike PS1-11af, none of the published SLSNe exhibiting these UV features had a prior epoch of spectroscopy lacking them.

The spectral evolution of the SLSNe reflects the decreasing photospheric temperature. As mentioned above, the relatively constant colors of PS1-11 af are different from the rapid redward evolution of most SNe. The hydrogen-poor SLSNe 2010gx and PS1-10bzj shown in Figure 6 demonstrate this behavior. Despite colors near maximum light that approach those seen in PS111 af, by $\sim 20 \mathrm{~d}$ after peak they are redder than PS1-11af is even at Day +100 . The SLSN 2008es is also an interesting object for comparison. At early times, the spectra had a very blue continuum with only weak He II $\lambda 4686$ emission (Gezari et al. 2009a). Only at later times did Balmer lines from the hydrogenrich ejecta become apparent as the object cooled (Miller et al. 2009; Gezari et al. 2009a). The cooling is apparent from the redward evolution of the color curve in Figure 6 away from the colors of PS1-11af.

This distinction points to a fundamental difference between PS1-11af and the SLSNe, so we discuss it in more detail. In the absence of other sources of energy input, adiabatic expansion of SN ejecta causes them to cool. This is especially true after maximum light, because the photon diffusion timescale becomes shorter than the expansion timescale of the ejecta (Arnett 1982), and thus SNe powered by energy sources that decline with time (such as radioactive ${ }^{56} \mathrm{Ni}$ or the spindown energy of magnetars) also cool after maximum light. Even SNe IIP, which have additional energy input from hydrogen recombination, cool and become redder on the plateau (e.g., Leonard et al. 2002).

A possible exception to these trends is provided by SNe IIn, where ongoing circumstellar (CSM) interaction supplies the additional energy necessary to slow the color evolution. There are few published UV light curves of SNe IIn, but we show the Swift $u-v$ color curve of SN 2007pk in Figure 6 (Pritchard et al. 2012). That object was initially very blue, but it rapidly became redder as the level of CSM interaction was insufficient to prevent the ejecta shell from cooling. A subset of SNe IIn exhibit much slower evolution, with long-lived CSM interaction causing the light curve to fade very slowly. We show the $U-V$ light curve of a prominent recent example of such an object, SN 2010j1, in Figure 6 (Zhang et al. 2012). Although the color evolves very slowly, it is $>1$ mag redder than PS1-11af.

In addition, CSM interaction that is sufficiently strong and long-lived to slow the light curve evolution should also be apparent from the spectra. The same CSM that provides the ongoing interaction luminosity in SNe IIn is ionized by the SN shock prior to the interaction and produces strong Balmer line emission (Chugai 2001), but such lines are not present in PS1-11af at any time. Some models for SLSNe can fit their light curves with shock breakout through a thick CSM shell, but those models predict fast declines after the peak if there is no additional CSM material outside of the initial shell. If the wind continues outside the initial shell, as is necessary for a 
slow decline rate, SN IIn-like emission lines would be expected (Chevalier \& Irwin 2011; Ginzburg \& Balberg 2012).

The lack of color evolution of PS1-11af also has implications for the interpretation of the SED that are inconsistent with the SN hypothesis. It is common in the literature to fit SN SEDs with a single-temperature $\mathrm{BB}$ and compute $T_{\mathrm{BB}}$ and the implied radius of a spherical emitting region $\left(R_{\mathrm{BB}}\right)$. Although $\mathrm{SN}$ photospheres recede through the ejecta in a comoving sense, the photospheric radii are physically expanding, so $R_{\mathrm{BB}}$ generally increases with time through maximum light and well beyond. The luminosity still decreases after the peak of the light curve because the cooling $T_{\mathrm{BB}}$ sufficiently compensates for the increase in $R_{\mathrm{BB}}$. $R_{\mathrm{BB}}$ only starts to decrease once the $\mathrm{SN}$ ejecta start to become optically thin and the $\mathrm{SN}$ begins the transition to the nebular phase. This is true for objects ranging from normal SNe II (e.g., Leonard et al. 2002) and Ib/c (Soderberg et al. 2008; Modjaz et al. 2009) to the SN IIn 2007pk (Pritchard et al. 2012) and even SLSNe (Miller et al. 2009; Gezari et al. 2009a; Quimby et al. 2011; Chomiuk et al. 2011; Lunnan et al. 2013; Inserra et al. 2013).

However, with $L_{\mathrm{BB}} \propto R_{\mathrm{BB}}^{2} T_{\mathrm{BB}}^{4}$, the approximately constant $T_{\mathrm{BB}}$ of PS1-11af implies that $R_{\mathrm{BB}}$ rises and falls with $L_{\mathrm{BB}}$ in a manner not seen in $\mathrm{SNe}$, as shown in the bottom panel of Figure 8 . In addition, the significantly higher $T_{\mathrm{BB}}$ of PS1-11af corresponds to significantly smaller $R_{\mathrm{BB}}$ at late times. $R_{\mathrm{BB}}$ for PS1-11af never became larger than $\sim 1.2 \times 10^{15} \mathrm{~cm}$, and fell to $\sim 6 \times 10^{14} \mathrm{~cm}$ on Day +60 . By contrast, the SLSNe have $R_{\mathrm{BB}}$ that grow with time to values an order of magnitude larger, approaching $10^{16} \mathrm{~cm}$ by $\sim 60 \mathrm{~d}$ after maximum light (Quimby et al. 2011; Chomiuk et al. 2011; Lunnan et al. 2013). A SN interpretation of PS1-11 af would have to explain not only why $R_{\mathrm{BB}}$ remains so small for a source with $L_{\mathrm{BB}}$ comparable to SLSNe, but how $R_{\mathrm{BB}}$ can fall after maximum light without $T_{\mathrm{BB}}$ cooling significantly or PS1-11af exhibiting any signs in the SED or spectra of the ejecta becoming optically thin in the transition to the nebular phase.

The compactness of $R_{\mathrm{BB}}$ for PS1-11af has other implications as well. The P-Cygni features of SNe form in the region exterior to the optically thick photosphere. In homologous expansion, the velocities of absorption features should agree with $R_{\mathrm{BB}}$ of the photosphere divided by the time since explosion, to within factors of order unity due to radiative transfer effects (e.g., Kirshner \& Kwan 1974). In addition, $R_{\mathrm{BB}}$ for SLSNe have been shown to increase with time at a rate approximately equal to the velocities measured from the absorption lines (Quimby et al. 2011; Chomiuk et al. 2011; Lunnan et al. 2013).

At the epoch of our Day +24 spectrum, we estimate that $R_{\mathrm{BB}}$ for PS1-11af was $9.2 \times 10^{14} \mathrm{~cm}$. This occurred $38.5 \mathrm{~d}$ after the first detection, which sets a lower limit on the time since explosion in a SN interpretation. In turn, this would imply that the material at the photosphere could be moving at a maximum of $2800 \mathrm{~km} \mathrm{~s}^{-1}$. However, the UV absorption features have FWHMs of $\sim 10^{4} \mathrm{~km} \mathrm{~s}^{-1}$, regardless of their identification. If we identify the $2680 \AA$ absorption as being due to $\mathrm{Mg}$ II $\lambda 2800$ doublet, the minimum of the absorption is blueshifted by $\sim 13,000 \mathrm{~km} \mathrm{~s}^{-1}$. In other words, material moving in homologous expansion at the velocities implied by the absorption features would be far away (radii $\gtrsim 4.3 \times 10^{15} \mathrm{~cm}$ ) from the inferred BB photosphere.

Finally, the host environment of PS1-11af is unlike that of any known hydrogen-poor SLSN. The host galaxies of all hydrogenpoor SLSNe studied to date have blue colors, strong emission lines, and other evidence of vigorous star formation activity (e.g., Neill et al. 2011; Quimby et al. 2011; Chomiuk et al. 2011; Stoll et al. 2011; Lunnan et al. 2013). Although we cannot exclude the possibility of an undetectably small amount of star formation in the local environment of PS1-11af around the host nucleus, the dominant stellar population is clearly older than a Gyr (Section 3.1).

In summary, $\mathrm{SNe}$ exhibit consistency between the various BB parameters and their time evolution with their spectroscopic features, due to the basic properties of expanding ejecta. However, the long-lasting blue colors of PS1-11af are evidence of a different time evolution driven by different underlying physics. The spectroscopic absorption features are not easy to accommodate in a model with approximately thermal ejecta in simple homologous expansion. The fits imply that $R_{\mathrm{BB}}$ does not expand and $T_{\mathrm{BB}}$ does not cool in the manner expected for $\mathrm{SN}$ ejecta. A SN interpretation for PS1-11 af would have to explain the apparently unique relationships between these observables for this object as well as its location at the center of a massive galaxy exhibiting no signs of recent star formation.

\section{PS1-11af AS A TDE}

Having ruled out SNe and AGN activity as plausible explanations for PS1-11af, we now interpret the observations in terms of a TDE. We estimate the expected black hole mass $\left(M_{\mathrm{bh}}\right)$ of the host of PS1-11af using scaling relations from the local universe. There is some ambiguity in the proper estimate for the bulge mass because we do not fully resolve potential substructure in the galaxy. If we identify the central $7 \times 10^{9} M_{\odot}$ core of the light profile with the bulge, the relations of Häring \& Rix (2004) predict $M_{\mathrm{bh}} \approx(8 \pm 2) \times 10^{6} M_{\odot}$, while using the total stellar mass of the galaxy likely sets an upper limit on $M_{\mathrm{bh}}$ of (1.6 \pm $0.4) \times 10^{7} M_{\odot}$. In the following discussion, we initially adopt $M_{\mathrm{bh}}=10^{7} M_{\odot}$, with a factor of two uncertainty.

\subsection{Optical Comparison to Previous TDEs}

PS1-10jh was a well-observed TDE discovered by PS1 (Gezari et al. 2012). It had a similar light curve shape to PS111 af (Figure 11), with similarly long-lived blue optical colors (Figure 6). However, the NUV to optical color was bluer for PS1-10jh, implying a minimum $T_{\mathrm{BB}}$ of $30,000 \mathrm{~K}$. Gezari et al. (2012) argue that the intrinsic $T_{\mathrm{BB}}$ had to be even higher, with a minimum of $\sim 50,000 \mathrm{~K}$ being necessary to supply sufficient ionizing photons for the observed He II emission lines at early times. A modest amount of reddening $(E(B-V)=0.08 \mathrm{mag})$ could reconcile this with the data.

We investigate the possibility of extinction for PS1-11af and find that $E(B-V) \approx 0.2$ mag is necessary for the best-fit $T_{\mathrm{BB}}$ of the Day + 10 GALEX+PS1 SED to equal $30,000 \mathrm{~K}$, and doubling that value pushes $T_{\mathrm{BB}}$ up to nearly $10^{5} \mathrm{~K}$. However, such a large extinction would imply that $L_{\mathrm{BB}}$ is $1.6 \times 10^{46} \mathrm{erg} \mathrm{s}^{-1}$, more than ten times the Eddington luminosity $\left(L_{\text {Edd }}\right)$ for a $10^{7} M_{\odot}$ black hole. If we assume that the maximum permitted $L_{\mathrm{BB}}$ is $\sim 3 L_{\mathrm{Edd}}$ for $M_{\mathrm{bh}} \approx 10^{7} M_{\odot}$ (the factor of three is to be conservative and allow for some uncertainty in the parameters), then we can set an upper limit on the reddening of $E(B-V) \lesssim$ $0.35 \mathrm{mag}$, which corresponds to an upper limit of $T_{\mathrm{BB}} \lesssim 5.4$ $\times 10^{4} \mathrm{~K}$. While the best fit for the host galaxy SED has zero reddening (Section 3.1), we cannot exclude the possibility of some gas and dust local to the environment of PS1-11af in the nucleus. The broadband SED fits for Sw 1644+ 57 implied substantially more extinction for the transient than was derived for the host galaxy (Bloom et al. 2011; Levan et al. 2011; 
Burrows et al. 2011; Zauderer et al. 2011). We note that none of our galaxy-subtracted spectra exhibit any narrow absorption lines from intervening gas (e.g., $\mathrm{Mg}$ II $\lambda 2800$, Ca II $\mathrm{H}+\mathrm{K}$, or $\mathrm{Na}$ I D), which argues against a large gas column along the line of sight.

The two optically selected SDSS TDEs of van Velzen et al. (2011a) were discovered on the decline, so there are only lower limits on the peak luminosities, but SDSS TDE2 appears to be most analogous to PS1-11af. It peaked at $L_{g}>4.1 \times$ $10^{43} \mathrm{erg} \mathrm{s}^{-1}$ and had an average $T_{\mathrm{BB}}=18,200 \mathrm{~K}$, both of which are fairly similar to PS1-11af. van Velzen et al. (2011a) identified several distinctive characteristics of their two events compared to other transients and variable AGN in Stripe 82, including the extremely blue color and slow luminosity and color evolution. They parameterized the slow evolution using the somewhat unusual units of $d \ln L_{g} / d t$ and $d \ln T_{\mathrm{BB}} / d t$. We estimate $L_{g}$ from $\lambda f_{\lambda}$ in $r_{\mathrm{P} 1}$ and $T_{\mathrm{BB}}$ from the BB fits to the PS1 $g_{\mathrm{P} 1} r_{\mathrm{P} 1} i_{\mathrm{P} 1} z_{\mathrm{P} 1}$ photometry above. We fit linear relationships to the logarithms of both of these quantities after maximum light and find $d \ln L_{g} / d t=(-1.8 \pm 0.2) \times 10^{-2} \mathrm{~d}^{-1}$ and $d \ln T_{\mathrm{BB}} / d t=$ $(-0.2 \pm 2) \times 10^{-3} \mathrm{~d}^{-1}$, very similar to the values for both of the SDSS TDEs.

van Velzen et al. (2011a) also noted the unusual combination of the very blue color and its slow evolution for their objects. Our photometry does not extend sufficiently blue to cover the SDSS $u^{\prime}$ band, so we cannot directly compare our measurements to the observer-frame quantities used by van Velzen et al. (2011a). However, the higher redshift of PS1-11af allows us to compensate somewhat for this. We de-redshift our Day -5 and +24 spectra to $z=0.2$ (similar to the SDSS TDEs) and use STSDAS/SYNPHOT ${ }^{20}$ in IRAF to synthesize observer-frame colors. The average colors of PS1-11af from the two shifted spectra are $u^{\prime}-g^{\prime}=-0.24 \mathrm{mag}$ and $g^{\prime}-r^{\prime}=-0.18 \mathrm{mag}$. We also fit a line to the $g_{\mathrm{P} 1}-z_{\mathrm{P} 1}$ points (fairly close to $u^{\prime}-r^{\prime}$ in the rest frame) from Figure 6 and find a slope of $(0.1 \pm 3) \times$ $10^{-3} \mathrm{mag} \mathrm{d}^{-1}$, consistent with no evolution. The combination of these colors and their stability over a long time baseline places PS1-11af in the same parts of parameter space as both of the SDSS TDEs in the diagrams of van Velzen et al. (2011a), and away from the $\mathrm{SNe}$ and AGN-like variables. This is further evidence that their objects are of a similar class as PS1-11af.

Cenko et al. (2012a) described the discovery of PTF 10iya, a fast evolving and UV bright nuclear flare. They modeled it as being the result of the early super-Eddington phase of accretion following the tidal disruption of a solar-type star by a $\sim 10^{7} M_{\odot}$ black hole. That object was very different from PS1-11af, despite a similar $T_{\mathrm{BB}}$. It had a somewhat higher peak luminosity ( $\gtrsim 10^{44} \mathrm{erg} \mathrm{s}^{-1}$, depending on the extinction correction) but declined very rapidly $\left(\sim 0.3\right.$ mag d $\left.^{-1}\right)$ compared to PS1-11af. PTF 10iya also had a bright associated X-ray source, with $L_{X} \approx 10^{44} \mathrm{erg} \mathrm{s}^{-1}$. Unfortunately, we have no constraints on the high-energy emission from PS1-11af.

The two relativistic TDEs, Sw 1644+57 and Sw 2058+05, have much more limited optical data (apparently because of high extinction in the case of Sw 1644+57: Bloom et al. 2011; Zauderer et al. 2011). Sw 2058+05 did have a slowly evolving $\mathrm{UV} /$ optical light curve, but $T_{\mathrm{BB}}$ had a lower limit of $6 \times 10^{4} \mathrm{~K}$ and $L_{\mathrm{BB}} \gtrsim 10^{45} \mathrm{erg} \mathrm{s}^{-1}$, indicating a significantly more luminous accretion event than for PS1-11 af with a bluer SED (Cenko et al. 2012b).

\footnotetext{
20 http://www.stsci.edu/institute/software_hardware/stsdas/synphot
}

Several UV-selected TDE candidates have been found in GALEX observations (Gezari et al. 2008, 2009b). These events had bluer SEDs than PS1-11af ( $\alpha \approx 1.1-1.4)$, and their inferred $T_{\mathrm{BB}}$ are correspondingly hotter. Single-temperature BB fits found $T_{\mathrm{BB}}$ in the range (4.4-12) $\times 10^{4} \mathrm{~K}$ (Gezari et al. $2009 \mathrm{~b}$ ), which is more comparable to the $\gtrsim 10^{5} \mathrm{~K}$ expectations for accretion disks near the tidal radius. The light curves for these objects are also generally consistent with a $t^{-5 / 3}$ decline, although the lack of data points on the rise allows for some freedom in the light curve fits.

The TDE candidate D3-13 (Gezari et al. 2008) may be instructive for PS1-11af. A Chandra detection at late times required $T_{\mathrm{BB}}>1.2 \times 10^{5} \mathrm{~K}$, while the $\mathrm{UV} /$ optical data at earlier times were better fit by a separate $T_{\mathrm{BB}} \sim 10^{4} \mathrm{~K}$ component in a two-temperature fit. The data were not taken simultaneously but may point to the existence of a hotter component that is not obvious in the optical data, even if it dominates the bolometric luminosity (Gezari et al. 2008).

\subsection{Light Curve Fits}

We use the bolometric light curve from Figure 11 (the observed $g_{\mathrm{P} 1}$ light curve multiplied by a bolometric correction to match the $L_{\mathrm{BB}}$ from the GALEX+PS1 SED fit on Day +10) to constrain the accretion properties of PS1-11af. Third-order polynomial fits to the data around peak give a maximum $L_{\text {bol }}$ of $(8.5 \pm 0.2) \times 10^{43} \mathrm{erg} \mathrm{s}^{-1}$, where the errors are derived by Monte Carlo resamplings of the observed light curve. This translates to $L_{\text {bol }} / L_{\text {Edd }} \approx 0.07_{-0.03}^{+0.13}$. Similarly, we perform a trapezoidal integration of the light curve to find the minimum total radiated energy of $(4.1 \pm 0.1) \times 10^{50}$ erg between the first and last detections. If we assume a radiative efficiency factor, $\eta \approx 0.1$, then the minimum necessary accreted mass onto the black hole is $M \approx 0.002(0.1 / \eta) M_{\odot}$. This is only a lower limit because it does not include the tail of the light curve at late times and, as we saw above, a small amount of extinction can dramatically increase the flux in the UV where the peak of the SED is, and we have few constraints on the SED shape. Still, the low value for $L_{\text {bol }}$ (and hence the accreted mass) is suggestive of a scenario involving only a partial disruption of a star.

The observed range of $R_{\mathrm{BB}}$ is $\sim(5-12) \times 10^{14} \mathrm{~cm}$ (Figure 8). These values are not straightforward to interpret in the TDE case because they were derived from the normalization of the BB fits by assuming a spherical emitting surface. The geometry of the TDE accretion flow could be quite different, with most models assuming that a disk dominates the UV/optical continuum unless reprocessing is invoked (Loeb \& Ulmer 1997; Lodato \& Rossi 2011; Strubbe \& Quataert 2011; Guillochon et al. 2013). Nevertheless, these radii provide a useful scale for the system, as they correspond to 135-400 Schwarzschild radii. Even allowing for uncertainty in $M_{\mathrm{bh}}$, they are far outside the expected tidal radius for a main sequence star. These radii are near the tidal radius for red giant stars, but TDEs with disruption radii that far from the central black hole are expected to have light curves with rise times of order a year, rather than a few weeks (MacLeod et al. 2012b).

We emphasize that these numbers have substantial systematic uncertainties. Most of the energy is emitted in the UV, where we only have the limited GALEX observations to constrain the SED and light curve, although $T_{\mathrm{BB}}$ determined from the PS1 observations alone is consistent with the fit to GALEX+PS1. Our assumption that the SED can be approximated as a singletemperature BB is definitely an oversimplification, as demonstrated by the UV excess of the galaxy-subtracted spectra 


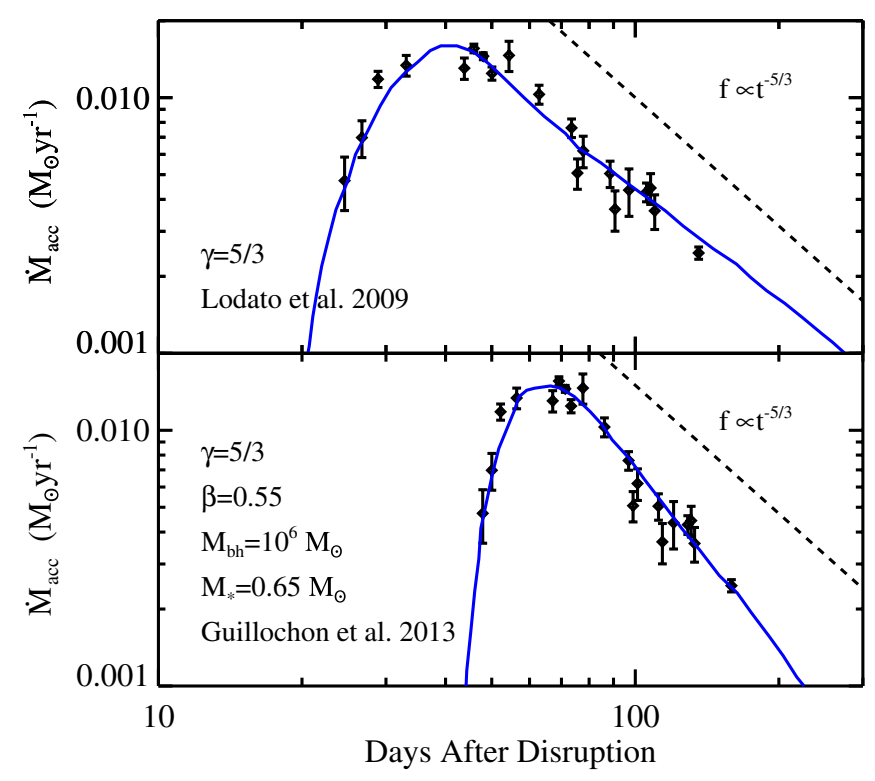

Figure 12. Fits of TDE models to $\dot{M}_{\text {acc }}$. We convert the bolometric light to $\dot{M}_{\text {acc }}$ by assuming a fiducial radiative efficiency factor of $\eta=0.1$. The top panel shows a fit using the $\gamma=5 / 3$ model of Lodato et al. (2009), with the time of disruption occurring $39 \mathrm{~d}$ before the peak of the light curve. The bottom panel uses the partial disruption model of Guillochon \& Ramirez-Ruiz (2013), with disruption occurring $62 \mathrm{~d}$ before peak. In each panel, the dashed line marks the traditional $t^{-5 / 3}$ power law expectation for TDE light curves.

(A color version of this figure is available in the online journal.)

relative to a $\mathrm{BB}$ (Figure 7). We know that some UV absorption features are present on Day +24 , near the time of the GALEX observations, so if any others were present in the NUV bandpass, the UV flux might be suppressed and $T_{\mathrm{BB}}$ would be underestimated.

We have no information about any possible emission extending to higher energies. In the cases of PTF 10iya and D3-13, the X-ray emission carried a comparable or greater amount of energy to the UV/optical component without lying on an extrapolation of the low-energy SED (Cenko et al. 2012a; Gezari et al. 2008). If such emission were present in PS1-11af, it would be undetectable in our dataset. Our derived values for $\dot{M}_{\text {acc }}$ are therefore best regarded as lower limits.

We now consider the shapes of the light curves to see if they are consistent with expectations from numerical modeling of stellar disruptions. We start with the estimated bolometric light curve from above and convert it to a mass accretion rate, $\dot{M}_{\text {acc }}$, assuming that $\eta=0.1$. The output $\dot{M}_{\text {acc }}$ of numerical simulations are self similar, with the time and accretion rate variables being rescalable functions of $M_{\mathrm{bh}}$ and the mass and radius of the disrupted star, $M_{\star}$ and $R_{\star}$.

We first fit to the simulations of Lodato et al. (2009), which were performed for the full disruption of a star at the tidal radius with a range of polytrope indices ( $\gamma$; assuming an equation of state with $\left.P \propto \rho^{\gamma}\right)$. We focus on models with $\gamma=5 / 3$, appropriate for low-mass stars, because of the small accreted mass for PS1-11af. We find an excellent fit (top panel of Figure 12) for a disruption occurring $39 \mathrm{~d}$ prior to the peak of the light curve if we scale the time variable from the fiducial value by $0.73 \pm 0.03$. With the scalings from Lodato et al. (2009), this implies $M_{\mathrm{bh}}=(5.4 \pm 0.5) \times 10^{5}\left(M_{\star} / M_{\odot}\right)^{2}$ $\left(R_{\star} / R_{\odot}\right)^{-3} M_{\odot}$. Even for a representative low-mass main sequence star with $M_{\star}=0.3 M_{\odot}$ (and $R_{\star}=0.3 R_{\odot}$; Tout et al. 1996), $M_{\text {bh }}$ is still $\sim 1.8 \times 10^{6} M_{\odot}$, well below our expectations from the host scaling arguments. Also, we had to apply a large vertical scaling factor to the plotted curve because $\dot{M}_{\text {acc }}$ is so low. The integral under the curve implies a total accreted mass of only $0.003 M_{\odot}$. This is inconsistent with the full disruption of even a low-mass star, which was one of the assumptions of the Lodato et al. (2009) simulations.

Therefore, we also examine partial disruption models (Guillochon \& Ramirez-Ruiz 2013), which parameterize the ratio of the tidal radius to the pericenter distance where the (partial) disruption occurs as $\beta$. Guillochon \& Ramirez-Ruiz (2013) give scaling relations for their families of curves in terms of several observables, but two of the parameters, the time from disruption to the peak and the asymptotic power-law index, depend on knowing the unobserved time of disruption. We estimate the mass lost by the star as $2 \times M_{\text {acc }} \approx 0.006 M_{\odot}$, from which we estimate $\beta \approx 0.57$. We fit our light curve with the $\beta=$ 0.55 model of Guillochon \& Ramirez-Ruiz (2013) in the bottom panel of Figure 12 and can find good fits with $M_{\star}=0.65 M_{\odot}$ and $M_{\mathrm{bh}}=10^{6} M_{\odot}$. This is not intended to be a complete search of all parameter space, but instead it is intended to demonstrate that the shape of the light curve is compatible with the curve of $\dot{M}_{\text {acc }}(t)$ from a partial-disruption event.

The fits with both sets of models prefer smaller values for $M_{\text {bh }}\left(\sim 10^{6} M_{\odot}\right)$ by about an order of magnitude than we expect based on the mass of the host galaxy. In each case, it is driven by the short rise time of the light curve because the time axis scales as $M_{\mathrm{bh}}{ }^{1 / 2}$. Our first detection of PS1-11af is on Day -14.5 at a flux level that is a factor of $\sim 3-4$ below the peak. By contrast, PS1-10jh had a rise time after the first detection of $\sim 50$ $\mathrm{d}$ (Figure 11; Gezari et al. 2012). This is not just a consequence of PS1-10jh being brighter and more easily detectable at fainter flux levels because it still took more than $30 \mathrm{~d}$ to rise the final two magnitudes to maximum light. Gezari et al. (2012) found that the same $\gamma=5 / 3$ model of Lodato et al. (2009) required a time stretch factor of 1.38 , compared to 0.73 for PS1-11af. Similarly, Guillochon et al. (2013) found a good fit to PS1-10jh with their partial disruption models with $\beta=0.87$ and $M_{\mathrm{bh}}=$ $10^{7} M_{\odot}$. Their derived $M_{\mathrm{bh}}$ was somewhat higher than expected from the host stellar mass relationship, which they attribute to scatter in the $M_{\mathrm{bh}}-M_{\text {bulge }}$ relationship.

A major problem with the interpretation of both fits to the PS1-11af light curve is that the connection between $\dot{M}$ of stellar debris returning to pericenter and the light curve in any given observed band depends on the details of the hydrodynamics of the gas (e.g., whether an outflow forms) and the radiative transfer, along with their evolution through the event. We use the observed constancy of the colors to estimate $\dot{M}_{\text {acc }}$ from the $g_{\mathrm{P} 1}$ light curve with a constant scaling factor; however, it is not clear that this is always justified and is certainly not expected in basic models.

Lodato \& Rossi (2011) computed multiband light curves for the Lodato et al. (2009) models, including the effects of a wind (Strubbe \& Quataert 2009). None of their models exceed $v L_{v}$ of $\sim$ few $\times 10^{42} \mathrm{erg} \mathrm{s}^{-1}$ in the optical band, an order of magnitude below that observed for PS1-11af, and most are closer to $10^{41} \mathrm{erg} \mathrm{s}^{-1}$. This is a consequence of the models having hotter spectra than PS1-11af and emitting more of their radiation at higher energy. In addition, the model spectra evolve strongly with time, leading to light curve shapes in each band that are quite different from the mass return rates derived from the properties of the disrupted stars. Gezari et al. (2012) also found that PS1-10jh had a light curve shape that closely matched the shape of the disruption models of Lodato et al. (2009) and lacked the expected color evolution. 
Guillochon et al. (2013) were able to fit the shapes of the light curves of PS1-10jh by including a reprocessing component to convert the accretion disk luminosity to a softer component with a roughly constant temperature, although their model invoked an unusually gray dust extinction law. They emphasize that pure accretion disk models cannot simultaneously satisfy the condition that the luminosity directly follows $\dot{M}_{\text {acc }}$ and maintain a constant color. The origin of the reprocessing material is not understood. It could result from shocks at the disruption radius as the returning stellar debris interacts with itself or it could represent a version of the accretion disk winds seen in regular AGN (Murray et al. 1995). Reprocessing of some form has long been invoked to explain AGN SEDs, which also exhibit low disk temperatures relative to the expectations of naive thin disk models (Koratkar \& Blaes 1999). See Lawrence (2012) for a recent review of this issue and some possible solutions.

Strubbe \& Quataert (2011) predict the existence of a TDE outflow, but only as long as the accretion rate is super-Eddington. After maximum light, as the rate of mass return to pericenter drops, the optical depth in the wind drops and leaves the hotter disk more exposed (Lodato \& Rossi 2011). $T_{\mathrm{BB}}$ for PS1-11af does not evolve in this fashion. For our inferred accretion rate for PS1-11af to approach Eddington would require invoking some UV extinction, an unobserved high-energy emission component (or at least more emission at shorter wavelengths than implied by the Wien tail of our single-temperature BB fits), or a smaller than expected $M_{\mathrm{bh}}$. In an outflow scenario, the slight decrease in $R_{\mathrm{BB}}$ after maximum light could be explained by the decrease in $\dot{M}_{\text {acc }}$ (and hence $L_{\mathrm{BB}}$ ) providing less radiation pressure and a weaker wind. Strubbe \& Quataert (2011) also include the effects of the unbound material from the disrupted star in their models, but the simulations of Guillochon et al. (2013) indicate that selfgravity confines the unbound material. It also has little effect on the SED or spectrum.

In summary, the shape of the light curve of PS1-11af can be acceptably fit by both full and partial tidal disruption models (Figure 12). However, the normalization to the low observed luminosity requires an accreted mass that is too low for a full disruption of a star. This inconsistency with the full disruption model leads us to favor a partial disruption scenario. However, if the bulk of the luminosity is emitted at higher energy, then the total accreted mass could be significantly higher. We note that the two models shown in Figure 12 have late-time decay rates that are in one case slower and in the other case faster than the canonical $t^{-5 / 3}$ value. This demonstrates the perils of attempting to match the observed late-time decay rate to theoretical expectations when the time of disruption is not observed, even for objects with light curves that are well sampled on the rise to maximum light.

\subsection{Lack of a Relativistic Jet}

Sw 1644+57 and Sw 2058+05 were both discovered due to a high-energy trigger and their long-lived, luminous X-ray counterparts were interpreted as the results of on-axis relativistic jets (Bloom et al. 2011; Levan et al. 2011; Burrows et al. 2011; Zauderer et al. 2011; Cenko et al. 2012b). We have no X-ray observations of PS1-11af, so we cannot exclude the possibility of such a high-energy counterpart here. However, any relativistic outflow should produce detectable radio emission, even if it is not oriented along our line of sight (Giannios \& Metzger 2011). Most radio observations to date of other TDEs and TDE candidates have not detected any emission (Bower et al. 2013; van Velzen et al. 2013). However, there are one or two

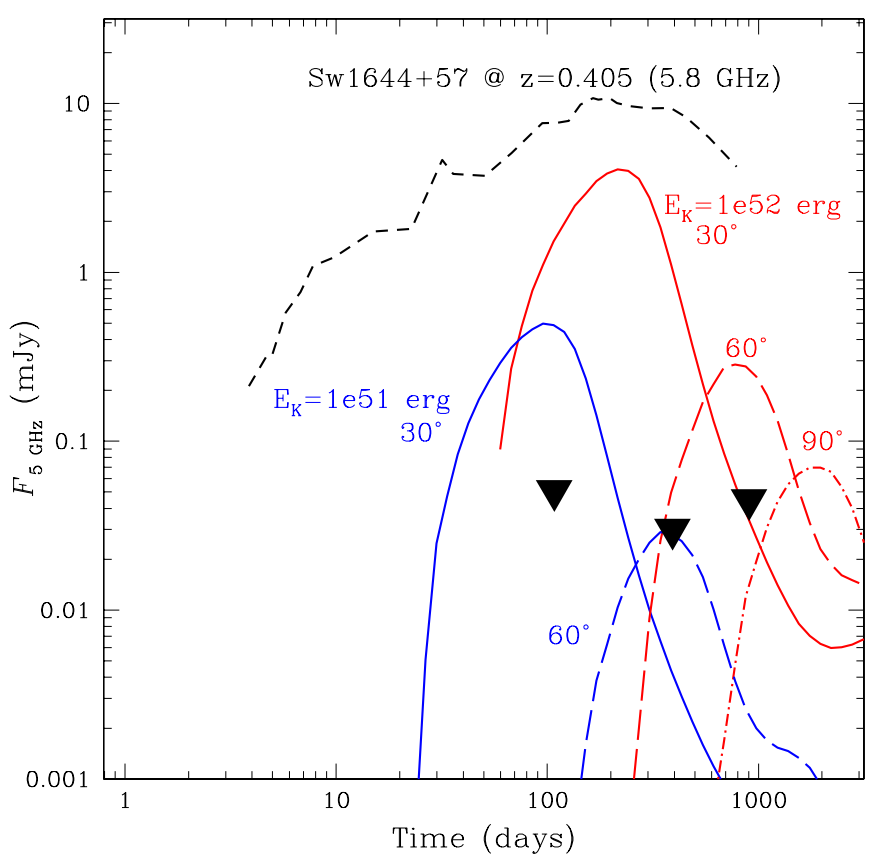

Figure 13. $5 \mathrm{GHz}$ upper limits for PS1-11af (black triangles). The abscissa represents the observer-frame time since disruption, which is assumed to be 40 $\mathrm{d}$ before the optical peak for PS1-11 af. The dashed line is the $5.8 \mathrm{GHz}$ light curve of Sw 1644+57 scaled to the redshift of PS1-11af (Zauderer et al. 2011, 2013; Berger et al. 2012). The other lines represent impulsive GRB-like afterglow models from the BOXFIT code (van Eerten et al. 2012), expanding into a constant density medium $\left(n=1 \mathrm{~cm}^{-3}\right)$ with an opening angle of $\theta_{\text {jet }}=0.1$. The solid and dashed lines in red have $E_{\mathrm{K}}=10^{52} \mathrm{erg}$ (as in Sw 1644+57; Zauderer et al. 2013), viewed $30^{\circ}$ and $60^{\circ}$ off-axis, respectively. The corresponding lines in blue have the kinetic energy scaled down by a factor of 10 . We also show a model (red dot-dashed line) with $E_{\mathrm{K}}=10^{52} \mathrm{erg}$ viewed $90^{\circ}$ off-axis (actually 89.95 for numerical reasons). In all models, the microphysical parameters representing the fractions of energy in the electrons and magnetic fields, $\epsilon_{\mathrm{e}}$ and $\epsilon_{\mathrm{B}}$, were fixed to match the best-fit values for $\mathrm{Sw} 1644+57$ of 0.1 and 0.01 , respectively (Zauderer et al. 2013).

(A color version of this figure is available in the online journal.)

$\mathrm{X}$-ray selected TDE candidates with late-time radio emission, potentially from off-axis jets (Bower et al. 2013).

Our three epochs of VLA non-detections strongly constrain the presence of any relativistic outflow. The redshift of PS111 af is only slightly higher than that of $\mathrm{Sw} 1644+57$, so an equivalently powerful relativistic jet would be easily detectable, even if it were viewed off-axis. As shown in Figure 13, the peak $5.8 \mathrm{GHz}$ radio flux of Sw 1644+57 (Zauderer et al. 2011; Berger et al. 2012) is a factor of 100-300 above our non-detections of PS1-11af on similar timescales.

Several models for the radio emission from jets produced by TDEs exist in the literature. One class invokes an analogy to gamma-ray burst (GRB) afterglows, with both reverse and forward shocks (Giannios \& Metzger 2011; Metzger et al. 2012) from the decelerating blast wave potentially contributing to the observed radio emission. An alternative invokes internal shocks within the jet, by analogy to AGN jets (van Velzen et al. 2011b). A relativistic outflow with a kinetic energy of $E_{\mathrm{K}}$ becomes nonrelativistic on a timescale of $\sim 300\left(E_{\mathrm{K}} / 10^{52} \mathrm{erg}\right)^{1 / 3} n^{-1 / 3} \mathrm{~d}$, where $n$ is the circumnuclear density, assumed to be uniform (e.g., Bower et al. 2013). On that timescale, comparable to that of our late-time observations of PS1-11af, the emission becomes more easily detectable by off-axis observers.

We take advantage of this fact to set limits on the presence of a relativistic jet by generating light curves using the GRB 
afterglow models produced by the BOXFIT code of van Eerten et al. (2012) rather than making detailed TDE jet models. The most important difference between jets produced by GRBs and by TDEs is that the former have a single impulsive episode of energy injection and the latter can have energy injection extending to late times (Berger et al. 2012; De Colle et al. 2012). However, we assume that this distinction primarily affects the shapes of the model light curves and is less important for predicting the timing and flux of the peak in the radio band, which mostly reflect the total energy in the jet and its orientation relative to our line of sight (van Eerten \& MacFadyen 2012).

We limit our models to those with microphysical parameters fixed to the best-fit values from afterglow models for $\mathrm{Sw}$ 1644+57 (Berger et al. 2012; Zauderer et al. 2013). BOXFIT assumes a constant density medium that we set to $n=1 \mathrm{~cm}^{-3}$. We initially use $E_{\mathrm{K}}=10^{52} \mathrm{erg}$ to match the total energy in the jet measured for $\mathrm{Sw} 1644+57$ for an assumed opening angle of $\theta_{\text {jet }}=0.1$ (Zauderer et al. 2013). Light curves observed $30^{\circ}$ and $60^{\circ}$ from the axis of such a jet are shown in Figure 13 and the peak fluxes clearly violate our upper limits for PS1-11af. If we scale the energy in the jet down by a factor of 10 , the $60^{\circ}$ off-axis case is only marginally consistent with the data. Also, a jet as powerful as Sw $1644+57$ oriented in the plane of the sky $\left(90^{\circ}\right.$ off-axis) could still be consistent with our limits because the radio peak is pushed to even later times (as expected; van Eerten \& MacFadyen 2012). An unusually low density medium could also suppress the radio emission. We defer more detailed consideration of the parameter space excluded by our limits on off-axis jet production to future work.

Jet formation in TDEs could be strongly tied to the accretion rate relative to Eddington (Giannios \& Metzger 2011; De Colle et al. 2012; Tchekhovskoy et al. 2013; van Velzen et al. 2013; Bower et al. 2013). By analogy with X-ray binaries, Tchekhovskoy et al. (2013) hypothesize that jets are features of either super-Eddington or strongly sub-Eddington phases. The jet in $\mathrm{Sw} 1644+57$ can be modeled by assuming that energy injection ended when the accretion rate dropped below a threshold value (Zauderer et al. 2013; De Colle et al. 2012). One possibility for PS1-11af not forming a jet is that the peak accretion rate of $\sim 0.07 \dot{M}_{\text {Edd }}$ simply never reached a sufficiently high value. If $M_{\text {bh }}$ really is closer to $10^{6} M_{\odot}$, as derived from the light curve fits, or if there is a significant unobserved highenergy emission component, then $\dot{M}_{\text {acc }}$ would be closer to the Eddington value and the lack of jet formation would have to be tied to some other parameter, such as the black hole spin or the magnetization of the stellar debris. Only $\sim 10 \%$ of optically luminous quasars are radio loud (Kellermann et al. 1989), and a similar fraction could be applicable to TDEs (Bower et al. 2013).

\subsection{Origin of Transient UV Absorption Features}

We now return to the most novel aspect of PS1-11af compared to previously observed TDEs and TDE candidates, the transient broad UV absorption features. A zoom-in on the two absorption features is shown in Figure 14. We fit Gaussians to the two absorption features in the galaxy-subtracted Day +24 spectrum. The shortest-wavelength one has a centroid of $2470 \AA$ and a FWHM of $10,100 \pm 1200 \mathrm{~km} \mathrm{~s}^{-1}$. The other one is centered at $2680 \AA$ with a FWHM of $10,200 \pm 400 \mathrm{~km} \mathrm{~s}^{-1}$. The rest-frame equivalent widths are $\sim 25$ and $50 \AA$, respectively. No additive offset has been applied to the flux scale in Figure 14, so the zeropoint is appropriate for the spectra. The $2680 \AA$ absorption has a minimum at about half of the interpolated continuum

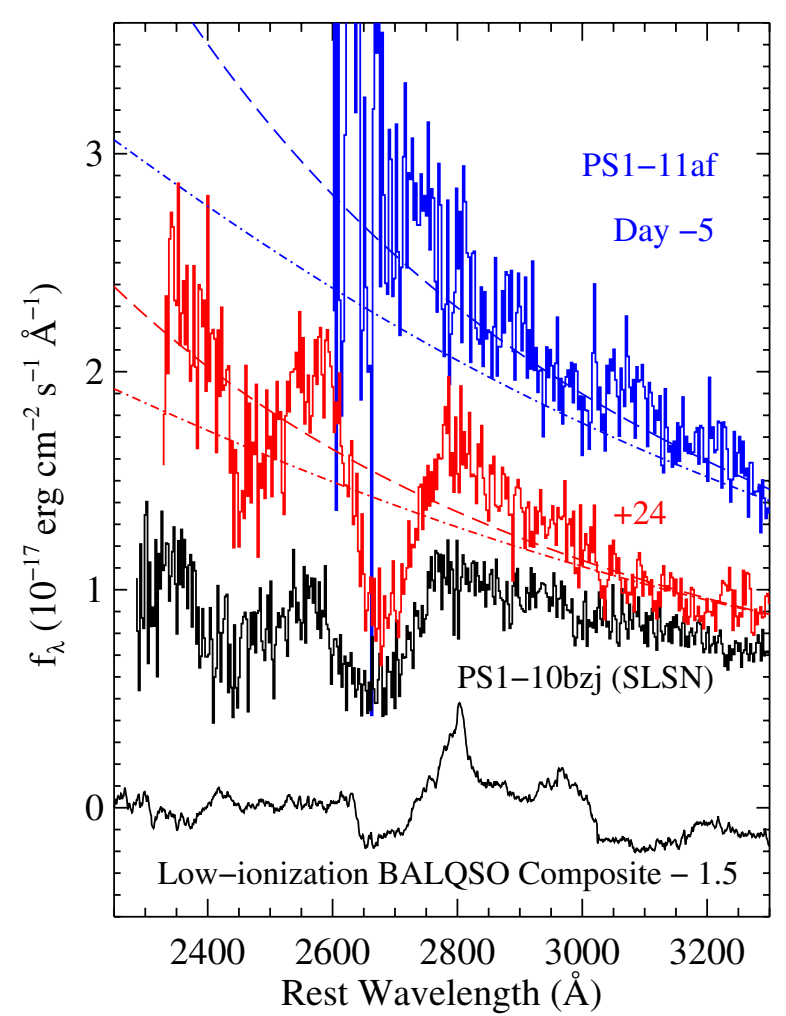

Figure 14. Broad UV absorption features in the Day +24 spectrum of PS1-11af (red). The Day -5 spectrum (blue) lacks any features. The top black spectrum is the same Day +7 spectrum of PS1-10bzj shown in previous plots (Lunnan et al. 2013). Although multiplicative scaling factors have been applied for clarity, no constant offsets have been added to the PS1-11af or PS1-10bzj spectra. Note that the PS1-10bzj spectrum exhibits narrow Mg II absorption from gas along the line of sight which is not present in either of the PS1-11af spectra. The bottom black spectrum is the composite low-ionization BALQSO spectrum from Brotherton et al. (2001), shifted down by the indicated amount. The dashed lines for each of the PS1-11 af spectra are power-law fits to the continuum between 3000-6000 ̊. The dot-dashed lines are $T_{\mathrm{BB}}=19,080 \mathrm{~K} \mathrm{BB}$ spectra normalized to match the continuum at $3500 \AA$ A. See Section 6.4 for discussion.

(A color version of this figure is available in the online journal.)

flux. Although the Day -5 LDSS spectrum becomes noisy very rapidly at shorter wavelengths due to the low instrument sensitivity in the blue, the $2680 \AA$ absorption would be quite prominent if it were present.

The redshift of PS1-11af is higher than most of the other TDEs, so the very few available spectra for other objects do not generally cover the wavelengths of these features, and we cannot determine whether they are present. For example, the spectra of PS1-10jh and SDSS TDE2 do not extend sufficiently far to the UV (Figure 10; Gezari et al. 2012; van Velzen et al. 2011a). An important exception is $\mathrm{Sw} 2058+05$, which is at the much higher redshift of 1.1853 and has no obvious broad spectral features in the rest-frame UV despite high-quality spectra (Cenko et al. 2012 b). The $2680 \AA$ feature would be on the blue edge of the observed wavelength range for the first spectrum of PTF 10iya, but it does not appear to be present (Cenko et al. 2012a).

PS1-11af also lacks the optical emission lines seen in some other objects. PS1-10jh exhibited broad He II $\lambda 4686$ and $\lambda 3203$ emission prior to maximum light, with FWHMs of 9,000 \pm $700 \mathrm{~km} \mathrm{~s}^{-1}$ (Gezari et al. 2012), and SDSS TDE2 had a broad $\mathrm{H} \alpha$ line after maximum light with a FWHM of $8000 \mathrm{~km} \mathrm{~s}^{-1}$ (van Velzen et al. 2011a). None of these lines appear in any of our PS1-11af spectra at any epoch, and it is clear from Figure 10 that we would have easily been able to detect a He II $\lambda 4686$ emission 
line with the same equivalent width as that seen in PS1-10jh. Gezari et al. (2012) argued that the observed SED of PS1-10jh did not supply enough ionizing photons to explain the observed He II line fluxes, and so invoked a modest amount of extinction and a higher intrinsic $T_{\mathrm{BB}}$. PS1-11af is not as blue as PS1-10jh, so one possibility is simply that it has a lower intrinsic $T_{\mathrm{BB}}$ and cannot ionize sufficient helium for detectable emission. It is also perhaps not a coincidence that the FWHMs of the absorption features in PS1-11af are similar to the widths of the emission features in the other objects (and in AGN) and may indicate that the line-forming regions are at similar distances from the central black hole (in units normalized to $M_{\mathrm{bh}}$ ).

We now consider possible identifications for these features. The red wing of the $2680 \AA$ absorption rejoins the apparent continuum level near $2800 \AA$ (Figure 14). The Mg II $\lambda 2800$ doublet is a very natural identification for this feature because it is strong in a wide variety of astronomical objects, and there are few strong isolated lines at nearby wavelengths. As stated in Section 5, the absorption minimum is blueshifted by $13,000 \mathrm{~km} \mathrm{~s}^{-1}$, a reasonable value given the FWHM of the feature. If the other feature is blueshifted by a similar amount, it implies that the rest wavelength of the absorbing ion should be near $2578 \AA$. One possible candidate is Fe II, which has strong resonance lines at $\lambda 2586$ and $\lambda 2600$. However, the feature at similar wavelengths seen in SLSNe such as our PS110bzj comparison has been identified as Si III $\lambda 2542$, following Quimby et al. (2011), and supported by detailed radiative transfer models (Dessart et al. 2012), although some Fe II may contribute to the blend (Lunnan et al. 2013).

Strubbe \& Quataert (2011) made predictions for spectral signatures of a wind produced by super-Eddington accretion at early times in TDEs. We inspect their output spectra, and the only line produced between 2500 and $3000 \AA$ in any of their models is Mg II. Their models with the Mg II absorption also predict stronger Balmer and optical He II absorption, which we do not observe. Their models assume a hot input spectrum $\left(T_{\mathrm{BB}} \gtrsim 10^{5} \mathrm{~K}\right)$ from the disk at the base of the wind leading to significantly hotter output spectra than we measure for PS111 af and hence a higher degree of ionization in their spectra. We conclude that the Mg II identification for the $2680 \AA$ absorption is robust, but we regard the other one as more uncertain.

Broad UV absorptions are seen in $\sim 10 \%$ of quasars, known as the BALQSOs (Weymann et al. 1991). Approximately $15 \%$ of these exhibit absorption in low-ionization lines, including the same Mg II and Fe II lines as those (possibly) present in PS1-11af (Voit et al. 1993). We show a composite spectrum of low-ionization BALQSOs (Brotherton et al. 2001) in Figure 14 as a comparison. This may be somewhat misleading, as the process of making a composite spectrum averages over discrete absorptions that are frequently narrower or detached from the rest wavelength. The typical shapes of the absorption troughs in BALQSOs (e.g., Voit et al. 1993; Hall et al. 2002) are generally quite different from the single broad absorption with a smooth profile for each line in PS1-11af. At lower luminosities, the intrinsic UV absorption lines in Seyfert 1 galaxies tend to be narrow and located at much lower velocities than the $\sim 10^{4} \mathrm{~km} \mathrm{~s}^{-1}$ we see here (Crenshaw et al. 1999).

Low-ionization BALQSOs are on average redder than normal quasars due to dust extinction in the absorbers (Sprayberry \& Foltz 1992; Brotherton et al. 2001), but PS1-11af is instead bluer than normal quasar SEDs. While the high-ionization absorption lines in BALQSOs may be formed coincident with the BLR (Murray et al. 1995), recent photoionization work has determined that the low-ionization absorbers are located several kpc from the central black hole (e.g., Moe et al. 2009). We conclude that although the absorption features in PS1-11af potentially come from similar ions as those seen in low-ionization BALQSOs, the physical situation is very different.

Assuming a virial equilibrium with $v^{2} \approx G M_{\mathrm{bh}} / R$ and $M_{\mathrm{bh}}=10^{7} M_{\odot}$, the expected typical velocities $(v)$ for material located near our derived $R_{\mathrm{BB}}$ on Day +24 are $12,000 \mathrm{~km} \mathrm{~s}^{-1}$. This is impressively close to the measured absorption blueshift of the $\mathrm{Mg}$ II line, given the uncertainties in $M_{\mathrm{bh}}$. There are also geometric uncertainties in the interpretation of $R_{\mathrm{BB}}$ in an accretion scenario and the factors of order unity involved in relating the absorption velocities to the virial velocity if they are formed in some sort of outflow with an asymptotic velocity that is a fraction of the escape speed. Still, this approximate equivalence, along with the fact that the line absorption is completely blueshifted from the rest wavelength, is evidence that the line formation region is in an outflow just outside of the continuum formation region.

Guillochon et al. (2013) argued that the lack of hydrogen lines in PS1-10jh can be explained by high ionization in the line-formation region. They analogize to reverberation mapping results from AGN (Blandford \& McKee 1982; Peterson et al. 2004), which imply that the typical distances of $\mathrm{H} \alpha$ and $\mathrm{H} \beta$ emission from the central black hole for AGNs with the continuum luminosity of PS1-10jh are larger than the outer radius of the debris disk from the disrupted star. Such an explanation is harder to understand here if our line identifications are correct. It is true that our maximum values for $R_{\mathrm{BB}}$ are $1.2 \times 10^{15} \mathrm{~cm}$ ( $\sim 0.5$ light-days), which is significantly closer than the typical $\mathrm{H} \beta$ lags of $\sim 10 \mathrm{~d}$ for AGN with continuum luminosities similar to that of PS1-11af (Peterson et al. 2004). However, the Mg II $\lambda 2800$ emissivity of BLR clouds closely tracks that of $\mathrm{H} \beta$ under a wide variety of density, ionizing flux, and ionizing SED assumptions (Korista et al. 1997), and empirically, the FWHMs of the two emission lines are identical (McLure \& Jarvis 2002). This implies that the formation regions for these two lines should be very similar. Our spectrum with the likely $\mathrm{Mg}$ II absorption lacks any evidence of $\mathrm{H} \beta$ in emission or absorption (and does not extend sufficiently far to the red to include $\mathrm{H} \alpha$ ). Furthermore, the Fe II emission (at least in the optical) has been shown to originate in the outer parts of the BLR, farther than the $\mathrm{H} \beta$ region (Barth et al. 2013), although the only available reverberation lag measurement for the same resonant UV1 Fe II multiplet that we likely see here suggests that it may form closer to the high-ionization C IV and Ly $\alpha$ lines (Maoz et al. 1993).

One possible way to understand the simultaneous appearance of $\mathrm{Mg}$ II and the lack of Balmer lines starts from the observation that our spectrum with the line features lacks strong $\mathrm{Mg}$ II emission as well (cf. the BALQSO composite in Figure 14). The balance between the emission and absorption parts of the profile depends on the optical depths and relative importance of scattering versus true absorption in a particular line, as well as the geometry and density profile of the emitting region. In pure scattering and a spherical geometry for an outflow, we might expect to see some SN-like P-Cygni emission as well as absorption if there is an outflow.

Strubbe \& Quataert (2011) instead argued that the optical depths of resonance lines in the TDE outflow will be small, and in contrast to the line-driven disk wind models for BALQSOs (e.g., Murray et al. 1995), the absorption region in TDE outflows will form close to the continuum photosphere, which limits 
the geometric extent of the emission region and the equivalent width of any emission. Although the spectra of PS1-11af lack a clear quasar-like $\mathrm{Mg}$ II emission line, there is weak evidence for some broad emission. In Figure 14, we fit power laws to the 3000-6000 A continua and extrapolate them to the UV (dashed lines). On Day -5 , the power law remains a decent fit at bluer wavelengths (with a little excess emission). However, on Day +24 , there appears to be some excess relative to the power law near 2600 and $2800 \AA$, to the red of each of the absorption lines, with the continuum returning to match the best fit power law near $2400 \AA$. This is only weak evidence because it is sensitive to our assumption about the intrinsic continuum shape. If true, it is also possible that some of the UV excess relative to a $\mathrm{BB}$ seen in Figure 7 is actually a superposition of numerous weak emission features, as in the "Little Blue Bump" of quasars.

Observationally, some SNe have suppressed Balmer P-Cygni features at early times when the ejecta are hot, even when the ejecta are known to be hydrogen rich. The SLSN 2008es, which we used above as comparison for the color evolution of PS1-11af, is a good example. The Balmer lines did not become distinct until $T_{\mathrm{BB}}$ dropped below $\sim 15,000 \mathrm{~K}$, below the temperatures measured for PS1-11af (Miller et al. 2009; Gezari et al. 2009a). Even in normal SNe II at very early times, a similar effect is present when $T_{\mathrm{BB}}$ is near $2 \times 10^{4} \mathrm{~K}$ and very steep density gradients in the outer ejecta lead to a very small line formation region relative to the continuum photosphere (Dessart et al. 2008), but such an explanation would not apply in a situation with deep absorptions, where the line formation region is likely extended. Nevertheless, perhaps it is possible for radiative transfer effects to suppress the hydrogen absorption below the expectations of Strubbe \& Quataert (2011), although more detailed work is needed to verify this.

\section{CONCLUSIONS}

We have presented observations of the UV-bright transient PS1-11af, which was discovered by PS1 and also detected by GALEX. The transient is coincident with the nucleus of a quiescent early type galaxy with no evidence for either AGN activity or star formation. PS1-11af was detected by PS1 for almost four months in the rest frame and had unusually blue colors the entire time. A BB fit to the GALEX+PS1 SED gave $T_{\mathrm{BB}}=19,000 \mathrm{~K}$, with little sign of evolution over the course of observations. The large amplitude of the transient, combined with the lack of variability of the host in other observing seasons and the very blue colors, are inconsistent with an AGN interpretation.

Multiepoch spectroscopy of PS1-11af at early times revealed several unusual features. At Day -5 , the spectra were completely featureless and were well fit by a power-law continuum with $f_{v} \propto v^{0.75}$. By Day +24 , two broad UV absorption features became apparent. These features are strikingly similar to the P-Cygni absorption features in the UV seen in some classes of SLSNe. However, the derived BB parameters for the SED of the transient are hard to accommodate in a $\mathrm{SN}$ interpretation. $R_{\mathrm{BB}}$ does not expand, and $T_{\mathrm{BB}}$ does not cool. Moreover, the apparent velocities of the absorption features are too high to correspond to material in homologous expansion at the photospheric radius implied by $R_{\mathrm{BB}}$.

The basic observables of the colors and optical luminosities, as well as the slow evolution of both, are comparable to values reported for the previous optically selected TDEs, PS1-10jh (Gezari et al. 2012) and the two from SDSS (van Velzen et al. 2011a). The slow evolution is unlike the fast-declining event PTF 10iya (Cenko et al. 2012a). We can fit the shape of the light curve with models for $\dot{M}_{\text {acc }}$ from TDEs (Lodato et al. 2009; Guillochon \& Ramirez-Ruiz 2013), but we set a lower limit on the accreted mass of $\sim 0.002 M_{\odot}$, which is indicative of a partial disruption event. We lack any constraints on emission shortward of the GALEX NUV band, which could substantially raise the inferred $\dot{M}_{\text {acc }}$. Our three epochs of non-detections from the VLA over the course of two years after the disruption set strong constraints on the existence of any relativistic outflow, even one that is off-axis.

The relatively low and constant $T_{\mathrm{BB}}$ measured for PS1-11af and PS1-10jh require that the majority of the optical light is reprocessed from the accretion disk, which would otherwise be much hotter and emit a spectrum that evolves with time (Strubbe \& Quataert 2009, 2011; Lodato \& Rossi 2011; Guillochon et al. 2013). Our observations do not constrain the structure of the reprocessing component, but the broad blueshifted UV absorption features point to an outflow. Outflows have been predicted for the early super-Eddington phase of TDEs (Strubbe \& Quataert 2009), but our basic measured parameters imply that the peak accretion rate for PS1-11af is sub-Eddington. This contradiction can be avoided if the majority of the luminosity is emitted at higher energies by another spectral component, if there is significant extinction, or if $M_{\mathrm{bh}}$ is substantially lower than predicted by local scaling relationships. The last of these possibilities is perhaps the most exciting, as TDEs offer the promise of being one of the few probes of $M_{\mathrm{bh}}$ in distant quiescent galaxies. Clearly, self-consistent models for the formation of the reprocessing component are necessary if we wish to confidently use the observed properties of TDEs to study quiescent black holes in distant galaxies.

However, much uncertainty remains in the interpretation of the TDE light curves. Future objects would benefit from simultaneous observations at higher energies (such as with Chandra) to constrain the relative contributions of the hotter emission from a disk and the reprocessing component deduced from the cooler $T_{\mathrm{BB}}$ seen at UV and optical wavelengths. The connection between these cooler optically elected TDEs such as PS1-11 af and previously reported soft X-ray and UV flares with $T_{\mathrm{BB}} \gtrsim 10^{5} \mathrm{~K}$ is unclear (e.g., Komossa \& Bade 1999; Gezari et al. 2009b).

Finally, it is interesting to consider the observable properties of PS1-11af at different redshifts. At $z \approx 0.2$, similar to previous optically selected TDEs, the broad UV absorption features would not be accessible to most ground-based spectrographs. The transient would then have a featureless blue optical spectrum and would be associated with the nucleus of an early type galaxy and thus would probably not arouse suspicions of being a SN. Conversely, at higher redshifts, as anticipated for objects in the LSST era, the UV features of PS1-11af would be more easily observable, possibly along with others at shorter wavelengths. If such an event occurred in a galaxy exhibiting nebular emission lines or other evidence of star formation, it seems likely that the transient would be confused with a SN. In such a scenario, careful consideration of the SED of the transient and its (lack of) evolution in the LSST colors would be necessary to discriminate between the two interpretations. At the lower spatial resolution of observations at those higher redshifts, the number of $\mathrm{SNe}$ with positions consistent with the nucleus is already higher than the expected TDE rate (Strubbe \& Quataert 2011), making the search for TDEs similar to PS1-11af in the LSST dataset challenging. 
We thank the staffs at PS1, Magellan, Gemini, the MMT, and the VLA for their assistance with scheduling and performing these observations. We acknowledge useful discussions with E. Ramirez-Ruiz, the assistance of T. Laskar with some of the MMT observations, and the help of A. Monson with FourStar data reduction. B.A.Z. is supported by an NSF Astronomy and Astrophysics Postdoctoral Fellowship under award AST1302954. This paper includes data gathered with the $6.5 \mathrm{~m}$ Magellan Telescopes located at Las Campanas Observatory, Chile. Some observations reported here were obtained at the MMT Observatory, a joint facility of the Smithsonian Institution and the University of Arizona. The Pan-STARRS1 Surveys (PS1) have been made possible through the contributions of the Institute for Astronomy, the University of Hawaii, the Pan-STARRS Project Office, the Max-Planck Society and its participating institutes, the Max Planck Institute for Astronomy, Heidelberg and the Max Planck Institute for Extraterrestrial Physics, Garching, the Johns Hopkins University, Durham University, the University of Edinburgh, Queen's University Belfast, the HarvardSmithsonian Center for Astrophysics, the Las Cumbres Observatory Global Telescope Network Incorporated, the National Central University of Taiwan, the Space Telescope Science Institute, the National Aeronautics and Space Administration under grant No. NNX08AR22G issued through the Planetary Science Division of the NASA Science Mission Directorate, the National Science Foundation under grant No. AST-1238877, and the University of Maryland. Some observations were obtained under Program ID GS-2011A-Q-29 (PI: Berger) at the Gemini Observatory, which is operated by the Association of Universities for Research in Astronomy, Inc., under a cooperative agreement with the NSF on behalf of the Gemini partnership: the National Science Foundation (United States), the National Research Council (Canada), CONICYT (Chile), the Australian Research Council (Australia), Ministério da Ciência, Tecnologia e Inovação (Brazil) and Ministerio de Ciencia, Tecnología e Innovación Productiva (Argentina). S.J.S. acknowledges funding from the European Research Council under the European Union's Seventh Framework Programme (FP7/20072013)/ERC grant agreement No. 291222. The National Radio Astronomy Observatory is a facility of the National Science Foundation operated under cooperative agreement by Associated Universities, Inc. R.P.K.'s work on SNe is supported in part by NSF grant AST-1211196. Partial support for this work was also provided by NSF grant AST-1009749 to J.T.. STSDAS is a product of the Space Telescope Science Institute, which is operated by AURA for NASA. Development of the BOXFIT code was supported in part by NASA through grant NNX10AF62G issued through the Astrophysics Theory Program and by the NSF through grant AST-1009863. Some of the computations in this paper were run on the Odyssey cluster supported by the FAS Science Division Research Computing Group at Harvard University.

Facilities: PS1 (GPC1), Magellan:Baade (IMACS, FourStar), Magellan:Clay (LDSS3), MMT (Blue Channel spectrograph), Gemini:South (GMOS-S), EVLA

\section{REFERENCES}

Ade, P. A. R., Aghanim, N., et al. 2013, arXiv:1303.5076

Ahn, C. P., Alexandroff, R., Allende Prieto, C., et al. 2012, ApJS, 203, 21

Allington-Smith, J., Breare, M., Ellis, R., et al. 1994, PASP, 106, 983

Arnett, W. D. 1982, ApJ, 253, 785

Bade, N., Komossa, S., \& Dahlem, M. 1996, A\&A, 309, L35

Barbary, K., Dawson, K. S., Tokita, K., et al. 2009, ApJ, 690, 1358

Barth, A. J., Pancoast, A., Bennert, V. N., et al. 2013, ApJ, 769, 128
Berger, E., Zauderer, A., Pooley, G. G., et al. 2012, ApJ, 748, 36

Blandford, R. D., \& McKee, C. F. 1982, ApJ, 255, 419

Bloom, J. S., Giannios, D., Metzger, B. D., et al. 2011, Sci, 333, 203

Bogdanovic, T., Cheng, R. M., \& Amaro-Seoane, P. 2013, arXiv:1307.6176

Bogdanović, T., Eracleous, M., Mahadevan, S., Sigurdsson, S., \& Laguna, P. 2004, ApJ, 610, 707

Bongiorno, A., Mignoli, M., Zamorani, G., et al. 2010, A\&A, 510, A56

Bower, G. C., Metzger, B. D., Cenko, S. B., Silverman, J. M., \& Bloom, J. S. 2013, ApJ, 763, 84

Brotherton, M. S., Tran, H. D., Becker, R. H., et al. 2001, ApJ, 546, 775

Bruzual, G., \& Charlot, S. 2003, MNRAS, 344, 1000

Burrows, D. N., Kennea, J. A., Ghisellini, G., et al. 2011, Natur, 476, 421

Cenko, S. B., Bloom, J. S., Kulkarni, S. R., et al. 2012a, MNRAS, 420, 2684

Cenko, S. B., Krimm, H. A., Horesh, A., et al. 2012b, ApJ, 753, 77

Chevalier, R. A., \& Irwin, C. M. 2011, ApJL, 729, L6

Chomiuk, L., Chornock, R., Soderberg, A. M., et al. 2011, ApJ, 743, 114

Chugai, N. N. 2001, MNRAS, 326, 1448

Crenshaw, D. M., Kraemer, S. B., Boggess, A., et al. 1999, ApJ, 516, 750

De Colle, F., Guillochon, J., Naiman, J., \& Ramirez-Ruiz, E. 2012, ApJ, 760,103

Dessart, L., Blondin, S., Brown, P. J., et al. 2008, ApJ, 675, 644

Dessart, L., Hillier, D. J., Waldman, R., Livne, E., \& Blondin, S. 2012, MNRAS, 426, L76

Dougherty, S. M., \& Perley, R. A. 2010, in ASP Conf. Ser. 438, The Dynamic Interstellar Medium: A Celebration of the Canadian Galactic Plane Survey, ed. R. Kothes, T. L. Landecker, \& A. G. Willis (San Francisco, CA: ASP), 421

Dressler, A., Hare, T., Bigelow, B. C., \& Osip, D. J. 2006, Proc. SPIE, 6269

Esquej, P., Saxton, R. D., Komossa, S., et al. 2008, A\&A, 489, 543

Evans, C. R., \& Kochanek, C. S. 1989, ApJL, 346, L13

Filippenko, A. V. 1982, PASP, 94, 715

Filippenko, A. V. 1997, ARAA, 35, 309

Fossati, G., Maraschi, L., Celotti, A., Comastri, A., \& Ghisellini, G. 1998, MNRAS, 299, 433

Gezari, S., Basa, S., Martin, D. C., et al. 2008, ApJ, 676, 944

Gezari, S., Chornock, R., Rest, A., et al. 2012, Natur, 485, 217

Gezari, S., Halpern, J. P., Grupe, D., et al. 2009a, ApJ, 690, 1313

Gezari, S., Heckman, T., Cenko, S. B., et al. 2009b, ApJ, 698, 1367

Gezari, S., Martin, D. C., Forster, K., et al. 2013, ApJ, 766, 60

Giannios, D., \& Metzger, B. D. 2011, MNRAS, 416, 2102

Ginzburg, S., \& Balberg, S. 2012, ApJ, 757, 178

Greisen, E. W. 2003, Information Handling in Astronomy-Historical Vistas, ed. A. Heck (Astrophysics and Space Science Library, Vol. 285; Dordrecht: Kluwer), 109

Guillochon, J., Manukian, H., \& Ramirez-Ruiz, E. 2013, arXiv:1304.6397

Guillochon, J., \& Ramirez-Ruiz, E. 2013, ApJ, 767, 25

Hall, P. B., Anderson, S. F., Strauss, M. A., et al. 2002, ApJS, 141, 267

Hao, L., Strauss, M. A., Fan, X., et al. 2005, AJ, 129, 1795

Häring, N., \& Rix, H.-W. 2004, ApJL, 604, L89

Hills, J. G. 1975, Natur, 254, 295

Ho, L. C. 2008, ARA\&A, 46, 475

Hodapp, K. W., Siegmund, W. A., Kaiser, N., et al. 2004, Proc. SPIE, 5489, 667

Hook, I. M., Jørgensen, I., Allington-Smith, J. R., et al. 2004, PASP, 116, 425 Inserra, C., Smartt, S. J., Jerkstrand, A., et al. 2013, ApJ, 770, 128

Kaiser, N., Burgett, W., Chambers, K., et al. 2010, Proc. SPIE, 7733, 12K

Kellermann, K. I., Sramek, R., Schmidt, M., Shaffer, D. B., \& Green, R. 1989, AJ, 98, 1195

Kennicutt, R. C., Jr. 1998, ARA\&A, 36, 189

Kirshner, R. P., \& Kwan, J. 1974, ApJ, 193, 27

Komossa, S., \& Bade, N. 1999, A\&A, 343, 775

Komossa, S., Halpern, J., Schartel, N., et al. 2004, ApJL, 603, L17

Komossa, S., Zhou, H., Wang, T., et al. 2008, ApJL, 678, L13

Koratkar, A., \& Blaes, O. 1999, PASP, 111, 1

Korista, K., Baldwin, J., Ferland, G., \& Verner, D. 1997, ApJS, 108, 401

Kriek, M., van Dokkum, P. G., Labbé, I., et al. 2009, ApJ, 700, 221

Lawrence, A. 2012, MNRAS, 423, 451

Leonard, D. C., Filippenko, A. V., Gates, E. L., et al. 2002, PASP, 114, 35

Levan, A. J., Tanvir, N. R., Cenko, S. B., et al. 2011, Sci, 333, 199

Li, L.-X., Narayan, R., \& Menou, K. 2002, ApJ, 576, 753

Lodato, G., King, A. R., \& Pringle, J. E. 2009, MNRAS, 392, 332

Lodato, G., \& Rossi, E. M. 2011, MNRAS, 410, 359

Loeb, A., \& Ulmer, A. 1997, ApJ, 489, 573

Lunnan, R., Chornock, R., Berger, E., et al. 2013, ApJ, 771, 97

MacLeod, C. L., Ivezić, Ž., Sesar, B., et al. 2012a, ApJ, 753, 106

MacLeod, M., Guillochon, J., \& Ramirez-Ruiz, E. 2012b, ApJ, 757, 134 
Magnier, E. 2006, in Proceedings of The Advanced Maui Optical and Space Surveillance Technologies Conference, ed. S. Ryan (Kihei, HI: The Maui Economic Development Board), E5

Maksym, W. P., Ulmer, M. P., Eracleous, M. C., Guennou, L., \& Ho, L. C. 2013, MNRAS, 435, 1904

Maoz, D., Netzer, H., Peterson, B. M., et al. 1993, ApJ, 404, 576

McLure, R. J., \& Jarvis, M. J. 2002, MNRAS, 337, 109

Metzger, B. D., Giannios, D., \& Mimica, P. 2012, MNRAS, 420, 3528

Miller, A. A., Chornock, R., Perley, D. A., et al. 2009, ApJ, 690, 1303

Modjaz, M., Li, W., Butler, N., et al. 2009, ApJ, 702, 226

Moe, M., Arav, N., Bautista, M. A., \& Korista, K. T. 2009, ApJ, 706, 525

Morrissey, P., Conrow, T., Barlow, T. A., et al. 2007, ApJS, 173, 682

Murray, N., Chiang, J., Grossman, S. A., \& Voit, G. M. 1995, ApJ, 451, 498

Neill, J. D., Sullivan, M., Gal-Yam, A., et al. 2011, ApJ, 727, 15

Pastorello, A., Smartt, S. J., Botticella, M. T., et al. 2010, ApJL, 724, L16

Perley, R. A., Chandler, C. J., Butler, B. J., \& Wrobel, J. M. 2011, ApJL, 739, L1

Persson, S. E., Barkhouser, R., Birk, C., et al. 2008, Proc. SPIE, 7014, 95P

Peterson, B. M., Ferrarese, L., Gilbert, K. M., et al. 2004, ApJ, 613, 682

Pringle, J. E., \& Rees, M. J. 1972, A\&A, 21, 1

Pritchard, T. A., Roming, P. W. A., Brown, P. J., et al. 2012, ApJ, 750, 128

Quimby, R. M., Aldering, G., Wheeler, J. C., et al. 2007, ApJL, 668, L99

Quimby, R. M., Kulkarni, S. R., Kasliwal, M. M., et al. 2011, Natur, 474, 487

Ramirez-Ruiz, E., \& Rosswog, S. 2009, ApJL, 697, L77

Rees, M. J. 1988, Natur, 333, 523

Rest, A., Scolnic, D., Foley, R. J., et al. 2013, ApJ, submitted (arXiv:1310.3828)

Rest, A., Stubbs, C., Becker, A. C., et al. 2005, ApJ, 634, 1103

Schlafly, E. F., \& Finkbeiner, D. P. 2011, ApJ, 737, 103

Schlegel, D. J., Finkbeiner, D. P., \& Davis, M. 1998, ApJ, 500, 525

Schmidt, G. D., Weymann, R. J., \& Foltz, C. B. 1989, PASP, 101, 713

Scolnic, D., Rest, A., Riess, A., et al. 2013, ApJ, submitted (arXiv:1310.3824)

Soderberg, A. M., Berger, E., Page, K. L., et al. 2008, Natur, 453, 469
Sprayberry, D., \& Foltz, C. B. 1992, ApJ, 390, 39

Stoll, R., Prieto, J. L., Stanek, K. Z., et al. 2011, ApJ, 730, 34

Stone, N., Sari, R., \& Loeb, A. 2013, MNRAS, 435, 1809

Storchi-Bergmann, T., Eracleous, M., Livio, M., et al. 1995, ApJ, 443, 617

Strubbe, L. E., \& Quataert, E. 2009, MNRAS, 400, 2070

Strubbe, L. E., \& Quataert, E. 2011, MNRAS, 415, 168

Stubbs, C. W., Doherty, P., Cramer, C., et al. 2010, ApJS, 191, 376

Tchekhovskoy, A., Metzger, B. D., Giannios, D., \& Kelley, L. Z. 2013, arXiv: 1301.1982

Tonry, J., \& Onaka, P. 2009, in Advanced Maui Optical and Space Surveillance Technologies Conference, Proceedings of the Advanced Maui Optical and Space Surveillance Technologies Conference, ed. S. Ryan (Kihei, HI: The Maui Economic Development Board), E40

Tonry, J. L., Stubbs, C. W., Lykke, K. R., et al. 2012, ApJ, 750, 99

Tout, C. A., Pols, O. R., Eggleton, P. P., \& Han, Z. 1996, MNRAS, 281, 257

Ulmer, A. 1999, ApJ, 514, 180

Urry, C. M., \& Padovani, P. 1995, PASP, 107, 803

Vanden Berk, D. E., Richards, G. T., Bauer, A., et al. 2001, AJ, 122, 549

van Eerten, H. J., \& MacFadyen, A. I. 2012, ApJ, 751, 155

van Eerten, H., van der Horst, A., \& MacFadyen, A. 2012, ApJ, 749, 44

van Velzen, S., Farrar, G. R., Gezari, S., et al. 2011a, ApJ, 741, 73

van Velzen, S., Frail, D. A., Körding, E., \& Falcke, H. 2013, A\&A, 552, A5

van Velzen, S., Körding, E., \& Falcke, H. 2011b, MNRAS, 417, L51

Voit, G. M., Weymann, R. J., \& Korista, K. T. 1993, ApJ, 413, 95

Wang, T.-G., Zhou, H.-Y., Komossa, S., et al. 2012, ApJ, 749, 115

Webb, W., \& Malkan, M. 2000, ApJ, 540, 652

Weymann, R. J., Morris, S. L., Foltz, C. B., \& Hewett, P. C. 1991, ApJ, 373, 23

Wilhite, B. C., Vanden Berk, D. E., Kron, R. G., et al. 2005, ApJ, 633, 638

Yang, C.-W., Wang, T.-G., Ferland, G., et al. 2013, ApJ, 774, 46

Zauderer, B. A., Berger, E., Margutti, R., et al. 2013, ApJ, 767, 152

Zauderer, B. A., Berger, E., Soderberg, A. M., et al. 2011, Natur, 476, 425

Zhang, T., Wang, X., Wu, C., et al. 2012, AJ, 144, 131 